\title{
Factores ambientales que influyen en la ictiofauna de la laguna La Mancha, sitio Ramsar, Golfo de México
}

\author{
Silvia Díaz-Ruiz ${ }^{*}$, Arturo Aguirre-León ${ }^{2}$, Erika Mendoza-Sánchez ${ }^{3}$
}

\& Ana Laura Lara-Domínguez ${ }^{4}$

1. Laboratorio Ictiología y Ecología Costera, Departamento de Hidrobiología, Universidad Autónoma MetropolitanaIztapalapa. San Rafael Atlixco 186, Col. Vicentina, Ciudad de México 09340, México; sdr@xanum.uam.mx

2. Laboratorio Ecología Costera y Pesquerías., Departamento El Hombre y su Ambiente, Universidad Autónoma Metropolitana-Xochimilco. El Hueso 1100, Col. Villa Quietud, Ciudad de México 04960, México; agla1269@correo.xoc.uam.mx

3. Laboratorio de Integridad Biótica. Facultad de Ciencias Naturales. Universidad Autónoma de Querétaro, Campus Aeropuerto. Carretera a Chichimequilla, Ejido Bolaños 76140 Querétaro, México; ika07mendozr3@hotmail.com

4. Red de Ecología Funcional, Instituto de Ecología, A.C., Carretera Antigua a Coatepec 351 El Haya Xalapa 91070 Veracruz, México; ana.lara@inecol.mx

* Correspondencia

Recibido 24-VII-2017. Corregido 06-IX-2017. Aceptado 25-IX-2017.

\begin{abstract}
Environmental factors influencing the ichthyofauna of the La Mancha lagoon, Ramsar site, Gulf of Mexico. La Mancha lagoon in the Gulf of Mexico, is a coastal system with Ramsar category because of its high biodiversity, many species of fish depend on it to complete their life cycles. In this study we analyzed the spatial and temporal variation of fish diversity, abundance and associations, and its relationship with physicochemical factors. Between April 2005 and January 2008, 95 daytime samplings were carried out at seven sites, where water characteristics and the composition of fish community were recorded. The system presented spatial-temporal variations of the physicochemical parameters, defining contrasting environments according to the local gradients. A total of 5974 fish individuals, belonging to 50 species, 39 genera and 25 families were collected; four species were new records for the system. The diversity and richness of species were high in the brackish environment and low in freshwaters areas. In September (rainy season) and November ("nortes" season), diversity $\left(\mathrm{H}^{\prime}=1.52\right)$ and richness $(\mathrm{D}=1.99)$ were highest. Fish abundance was greater towards the mouth connecting with the sea. The density and biomass $\left(1.17 \mathrm{ind} / \mathrm{m}^{2}, 4.33 \mathrm{~g} / \mathrm{m}^{2}\right)$ was higher in April (dry season) and the average weight (30.82 g/ind) in July (rainy season). The Importance Value Index (IVI) defined four dominant species representing $75 \%$ of the numerical abundance and $46 \%$ of the total catch in weight. The Canonical Correspondence Analysis (CCA) showed that the fish-habitat relationship was explained in $57 \%$ of the total variance for the first two axes, where the variations in temperature, salinity, dissolved oxygen and depth were the environmental factors that determined the composition, distribution, community components and trophic categories of the fish community. This study provides information on the relationship between the environment and the structure of the fish community in La Mancha, and represents a basis for ecological monitoring to increase the knowledge needed about the ecosystem and fish populations, in order to boost management strategies for the fishery resources conservation in Veracruz. Rev. Biol. Trop. 66(1): 246-265. Epub 2018 March 01.
\end{abstract}

Key words: physicochemical factors, community structure, dominance, trophic structure, fish-habitat relationships, La Mancha, Mexico.

Las lagunas costeras del Estado de Veracruz en el Golfo de México se caracterizan por su alta biodiversidad y valor biológico, e incluyen una gran variedad de recursos pesqueros, ocupando el cuarto lugar en la producción pesquera nacional (Lara-Domínguez et al., 2011). En particular, el sistema lagunar La Mancha declarado sitio RAMSAR (Ficha Informativa 
de los Humedales de Ramsar, 2003) es pequeño y somero; presenta una conexión efímera con el mar la cual se cierra durante un periodo del año y se abre durante la época de lluvias, lo que permite el intercambio de organismos, nutrientes y agua. Este ecosistema lagunar, destaca por su alta producción primaria y riqueza de recursos biológicos, y es utilizado por una gran variedad de peces de manera temporal o permanente para llevar a cabo parte de sus ciclos de vida, como área de alimentación, crianza, desove y refugio, al mismo tiempo, representan especies para consumo local (Lara-Domínguez, Day, Yáñez-Arancibia, \& Sainz-Hernández, 2006; Díaz-Ruiz, Aguirre-León, Juárez, Matsumoto, \& Hernández, 2008). De esta manera, la utilización de los hábitats en estos sistemas por los peces, está relacionado con las variaciones de salinidad y temperatura, la vegetación circundante, la descarga de los ríos y el intercambio de agua con el mar, entre otras características, lo que influye en la estacionalidad de la diversidad, distribución, abundancia y frecuencia de las especies que habitan este sistema (Able, 2005; Barletta, Barletta-Bergan, Saint-Paul, \& Hubold, 2005; Díaz-Ruiz, Aguirre-León, Calva-Benítez, \& Barba-Macías, 2012; Zárate-Hernández, Castillo-Rivera, SanvicenteAñorve, \& Ortiz-Burgos, 2012; Aguirre-León, Pérez-Ponce, \& Díaz-Ruiz, 2014).

Adicionalmente, los peces funcionan como reguladores energéticos en la comunidad, al transformar, intercambiar y almacenar la energía, a través de complejas interacciones biológicas (e.g. reproducción, desove, alimentación, crecimiento desde el mar hacia los hábitats lagunares), que están relacionadas con los patrones de migración y el uso diferencial de los hábitats, como respuesta a la heterogeneidad ambiental del sistema (Deegan et al., 1986; Castillo-Rivera, Zavala-Hurtado, \& ZárateHernández, 2002; Arceo-Carranza, Vega-Cendejas, Montero, \& Hernández de Santillana, 2010; Aguirre-León et al., 2014).

No obstante, a pesar de la importancia ecológica y económica de los recursos pesqueros de la laguna La Mancha, la información sobre la estructura ecológica de la comunidad de peces en este sitio es escasa. Por lo tanto, el conocimiento sobre los procesos ambientales y su influencia en la estructura ecológica de los peces de este sistema lagunar es relevante, toda vez que no existen antecedentes de estudios a largo plazo en esta región. Los estudios en este sistema sólo incluyen listas de especies, como el de Reséndez-Medina y Kobelkowsky (1991) y sobre evaluación ecológica de Díaz-Ruiz et al. (2008). Por lo tanto, el presente estudio representa la primera información sobre la ictiofauna del sistema lagunar con un enfoque de estructura ecológica. Para este fin, se plantearon los siguientes objetivos: 1) analizar el comportamiento fisicoquímico del sistema en escala espacial y temporal, 2) determinar la composición de la ictiofauna, 3) evaluar los principales patrones de diversidad, abundancia y conjuntos de peces, con relación a las principales variables fisicoquímicas, y 4) definir la dominancia, componentes comunitarios y niveles tróficos de las especies de peces.

\section{MATERIALES Y MÉTODOS}

Área de estudio: La Laguna de La Mancha se localiza en la planicie costera central de Veracruz, en el Golfo de México (19³4'$\left.19^{\circ} 36^{\prime} \mathrm{N} \& 96^{\circ} 22^{\prime}-96^{\circ} 24^{\prime} \mathrm{W}\right)$. Tiene una superficie de $1.5 \mathrm{~km}^{2}$ y está integrada por dos cuerpos de agua unidos por un canal estrecho (Fig. 1). Es una laguna somera con profundidad promedio de $0.60 \mathrm{~m}$ en el norte a $2.5 \mathrm{~m}$ en el sur. Tiene influencia marina a través de una boca de conexión efímera que se abre en época de lluvias, y se cierra durante la época seca. Por la boca cruza un gasoducto, lo que ha modificado el intercambio de agua con el mar (LaraDomínguez et al., 2006). Recibe la descarga de los ríos Caño Grande y Sábalo en el extremo sur (Fig. 1). El clima es cálido subhúmedo con lluvias en verano; la temperatura media anual fluctúa entre 22 y $26{ }^{\circ} \mathrm{C}$ y la precipitación entre 1200 y 1500 mm anuales (García, 2004). Se reconocen tres épocas climáticas: secas (marzo a junio), lluvias (julio a octubre) y nortes (noviembre a febrero) (Contreras-Espinosa, Castañeda-López, \& Rivera-Guzmán, 2006). 


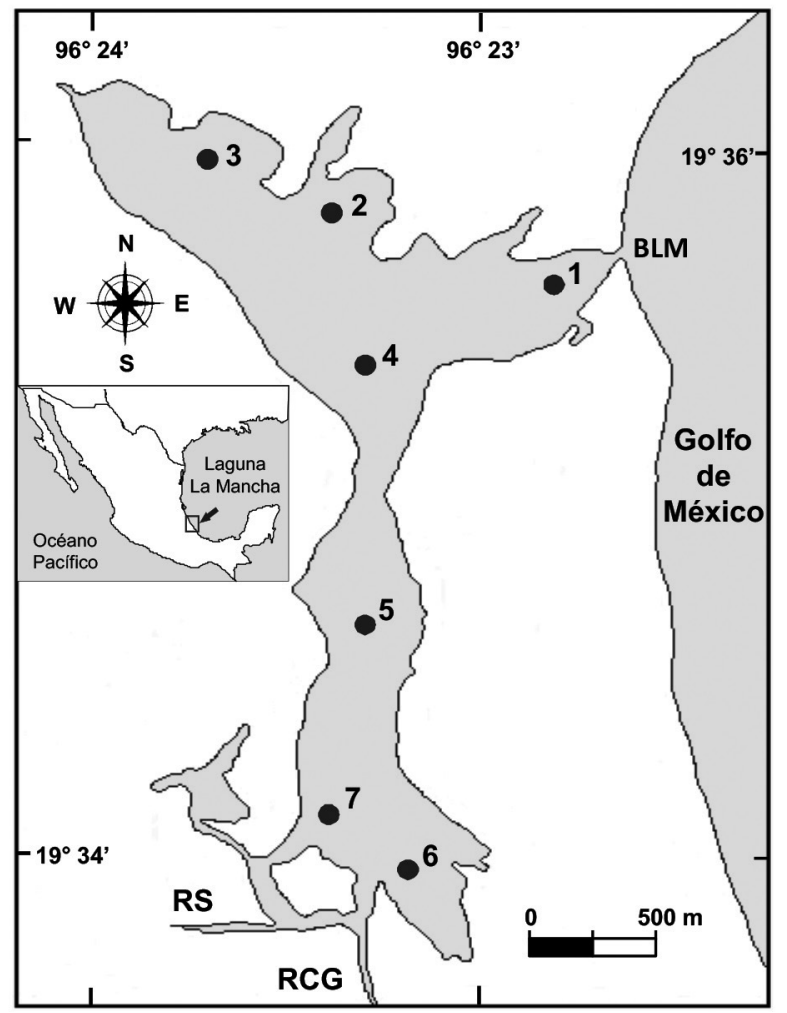

Fig. 1. Sistema lagunar La Mancha, Veracruz. Ubicación de estaciones de muestreo $(\bullet)$, fisiografía y ríos que drenan al sistema. Boca La Mancha (BLM), Río Caño Grande (RCG), Río Sábalo (RS).

Fig. 1. La Mancha Lagoon System, Veracruz. Location of sampling stations $(\bullet)$, physiographic of the study area and rivers draining into the system are noted. La Mancha Inlet (BLM), Caño Grande River (RCG), Sábalo River (RS).

Existen diferentes ambientes acuáticos en el sistema con vegetación circundante de manglar (Rhizophora mangle), sumergida (Rupia maritima, Siringodium filiforme) y áreas de alta sedimentación (Lara-Domínguez et al., 2006).

Actividades de campo y laboratorio: $\mathrm{La}$ recolección de peces se realizó en siete estaciones de muestreo durante dieciséis meses, entre abril 2005 y enero 2008 (Fig. 1). Los muestreos se realizaron cada tres meses en el 2005, y cada 2 meses en 2006 y 2007, incluyendo enero del 2008. Las capturas fueron diurnas (09:0015:00 horas), y en cada estación se efectuó un arrastre con un chinchorro playero de $50 \mathrm{~m}$ de largo, (caída de $2 \mathrm{~m}$, con luz de malla de $1.9 \mathrm{~cm}$ en el copo y $1.5 \mathrm{~cm}$ en las alas), y un área de $400 \mathrm{~m}^{2}$. Cada arrastre se llevó a cabo entre 20 a 30 minutos de maniobra, con un total de 95 capturas. Todas las muestras fueron fijadas en formaldehído al $4 \%$ neutralizado con borato de sodio, y posteriormente preservadas en etanol al $70 \%$ para su análisis en el laboratorio.

En cada muestreo, se registró la salinidad, temperatura y oxígeno disuelto, con un multianalizador YSI-3800; el pH con un potenciómetro Hanna-9812; la profundidad con una sonda graduada $(\mathrm{cm})$ y la transparencia con un disco de Secchi $(\mathrm{cm})$.

En el laboratorio se midió la longitud total (LT) de los peces con un ictiómetro graduado (cm) y se pesaron con una balanza digital Sartorius-Entris 2202-1S (precisión 0.01g). La identificación taxonómica de los peces se realizó con claves del Atlántico occidental y del Golfo de México (Fischer, 1995; Castro Aguirre, 
Espinoza-Pérez, \& Schmitter-Soto, 1999). El estatus taxonómico se basó en el criterio de Nelson (2006) y para corroborar la autoridad y nomenclatura actual de las especies, se utilizó FishBase (Froese, \& Pauly, 2017).

Se calcularon los parámetros ecológicos de la comunidad: diversidad $\mathrm{H}^{\prime}$ (Shannon, \& Weaver, 1963), riqueza de especies D (Margalef, 1969) y equitatividad J' (Pielou, 1966), así como la densidad (ind. $/ \mathrm{m}^{2}$ ), biomasa (g/ $\mathrm{m}^{2}$ ) y peso promedio de los peces (g/ind.) (Aguirre-León et al., 2014). La dominancia de las especies se determinó mediante el Índice de Valor de Importancia (IVI) utilizando el programa ANACOM (De la Cruz-Agüero, 1994). Para determinar el tipo de componente comunitario al cual pertenece cada especie, se siguió el criterio de Yáñez-Arancibia (1986), quien las ubica según su permanencia temporal en ocasional, cíclica o permanente en los sistemas estuarinos: $\mathrm{E}=$ estuarino, $\mathrm{ME}=$ marino eurihalino, $\mathrm{ES}=$ marino estenohalino, $\mathrm{D}=$ dulceacuícola. Se definió la categoría trófica de cada especie, analizando la información publicada sobre la alimentación y de acuerdo con el criterio propuesto por Yáñez-Arancibia et al. (1985): a) Consumidores de primer orden, son herbívoros exclusivos, fitoplanctófagos y/o zooplanctófagos, peces detritívoros y omnívoros, b) Consumidores de segundo orden, peces predominantemente carnívoros, pueden incorporar en su dieta restos vegetales y detritus, c) Consumidores de tercer orden, peces exclusivamente carnívoros, donde los vegetales y el detritus es un alimento accidental.

Para estimar las diferencias estadísticas espaciales (entre estaciones) y temporales (entre meses y años) de los parámetros físicoquímicos y parámetros ecológicos de la comunidad, se evaluó previamente la homogeneidad de varianzas entre grupos, con la prueba de Levene (1960); en los casos necesarios, se hicieron transformaciones logarítmicas. Cuando se cumplió la homogeneidad de varianzas se utilizó una prueba ANOVA-F-, cuando no se cumplió, fue utilizada la prueba Kruskal-Wallis-H- (Zar, 1999). Para todas las pruebas anteriores se consideró un nivel de significancia $\mathrm{p}<0.05$ (Zar, 1999; Hair, Anderson, Tatham, \& Black, 2001). Los análisis se realizaron con ayuda del paquete estadístico STATISTICA, versión 10. Se evaluó la relación entre las especies de peces y las variables ambientales del sistema, con un análisis de correspondencia canónica (ACC) (Ter Braak, \& Verdonschot, 1995). Para este fin, se utilizó una matriz de abundancia de las especies y de datos físicoquímicos por estación y por mes. Las correlaciones inter-set determinaron las variables ambientales que fueron más importantes en el comportamiento de las abundancias de las especies (McGarigal, Cushman, \& Stafford, 2000). El ACC fue generado usando el programa Multivariate Statistical Package (MSVP) versión 3.22.

\section{RESULTADOS}

Ambiente-escala espacial: El análisis espacial de los físicoquímicos de 2005 a 2007 2008 , mostró gradientes con valores bajos en la zona sur (estaciones 5 a 7), y altos en la zona norte del sistema (estaciones 1 a 4). El valor promedio más bajo de salinidad (11.8 ups \pm 12.2) se registró en la estación 7 del 2005 y el más alto (31.3 ups \pm 9.1$)$ en la estación 1 del 2007-2008 (Fig. 2A a C). Este parámetro sólo indicó diferencias significativas entre estaciones en 2007-2008 (ANOVA-F $=2.7$, $\mathrm{p}<0.05)$. La temperatura del agua presentó el valor promedio más bajo $\left(26.0{ }^{\circ} \mathrm{C} \pm 4.10\right)$ y más alto $\left(31.6{ }^{\circ} \mathrm{C} \pm 3.9\right)$ en la estación 6 durante 2005 y 2007-2008, respectivamente (Fig. 2D a F). El ANOVA no señaló diferencias significativas entre estaciones $(p>0.05)$. Un comportamiento similar a la salinidad lo presentó el oxígeno disuelto, con el valor promedio más bajo $(2.4 \mathrm{mg} / \mathrm{L} \pm 1.21)$ en la estación 7 del 2005 y el más alto $(5.7 \mathrm{mg} / \mathrm{L} \pm 0.97)$ en la estación 1 del 2006 (Fig. 2G a I). Este parámetro mostró diferencias significativas entre sitios en 2006 (ANOVA- $F=1.6, p<0.05$ ) y en 2007 (Kruskal Wallis-H = 7.8, p < 0.05). El valor promedio más bajo de $\mathrm{pH}(7.1 \pm 0.7) \mathrm{se}$ registró en la estación 6 del 2006, mientras que el más alto $(7.7 \pm 0.6)$ en la estación 1 del 2005 

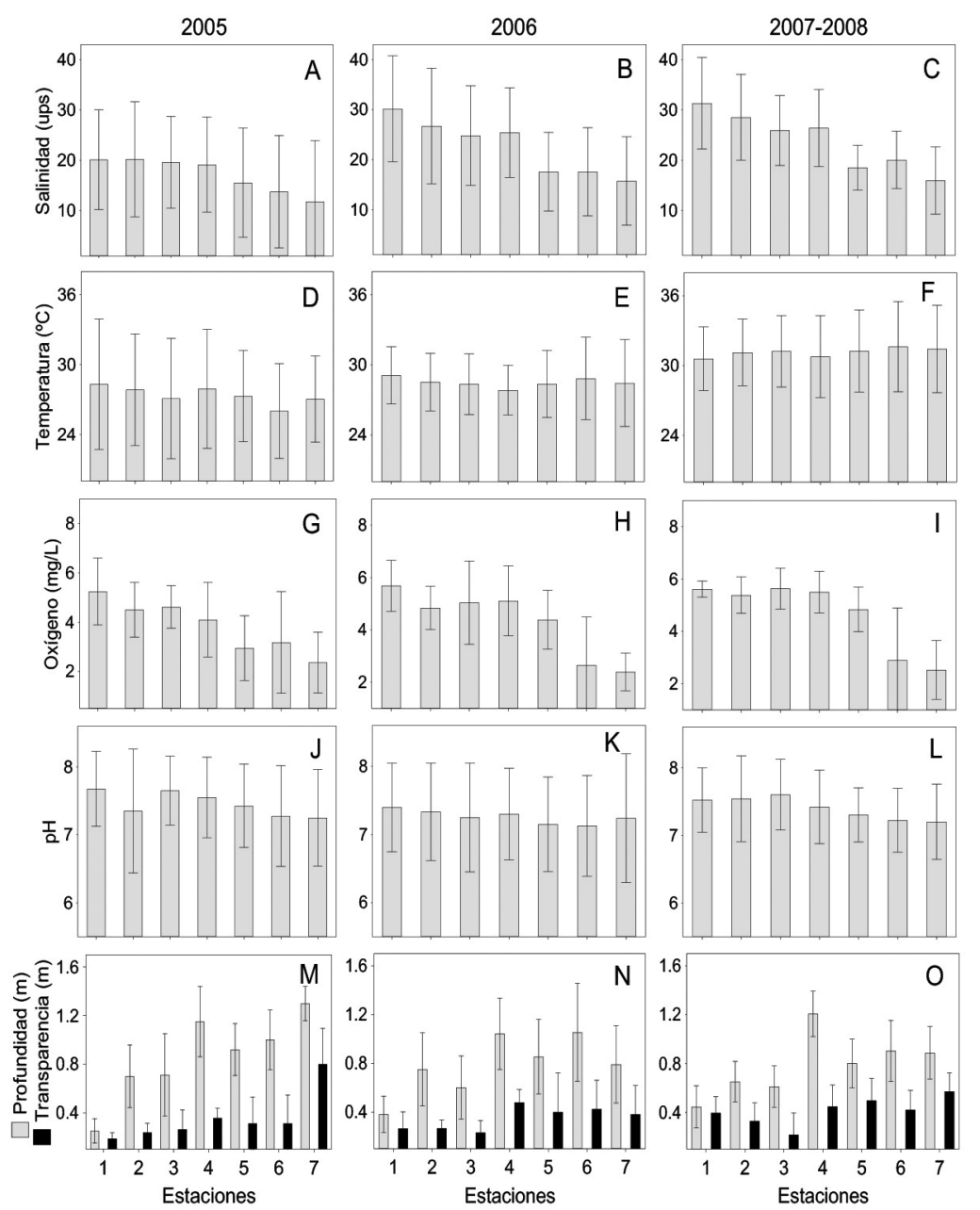

Fig. 2. Variación espacial de los parámetros fisicoquímicos en el sistema lagunar La Mancha.

Fig. 2. Spatial variation of the physicochemical parameters in the La Mancha Lagoon System.

(Fig. 2J a L), sin diferencias significativas entre sitios $(p>0.05)$. Un gradiente inverso a los parámetros anteriores se observó en la profundidad con el valor promedio más bajo $(0.25$ $\mathrm{m} \pm 0.1)$ en la estación $1 \mathrm{y}$ el promedio más alto $(1.3 \mathrm{~m} \pm 0.14)$ en la estación 7 del 2005 (Fig. 2M a O). Así, la transparencia más baja (31\%, valor promedio $0.8 \mathrm{~m} \pm 0.29$ ) se registró en zonas profundas (estación 6) del 2005, mientras que la más alta $(88 \%$, valor promedio $0.19 \mathrm{~m} \pm 0.05$ ) en zonas someras (estación 1) del 2007-2008 (Fig. 2M a O). La profundidad presentó diferencias significativas entre sitios en 2005 (ANOVA-F $=0.05, \mathrm{p}<0.05), 2006$
$($ ANOVA-F $=0.08, \mathrm{p}<0.05)$ y $2007-2008$ (ANOVA-F $=0.03, \mathrm{p}<0.05)$ y la transparencia en 2005 (Kruskal Wallis- $\mathrm{H}=10.3, \mathrm{p}<0.05$ ) y 2007-2008 (ANOVA-F=0.02, $\mathrm{p}<0.05$ ).

Ambiente-escala temporal: El valor promedio de salinidad más bajo (3.9 ups \pm 2.4 ) se presentó en julio 2005, mientras que el más alto (34.3 ups \pm 3.7 ) en septiembre del 2007 (Fig. 3A a C). Este parámetro mostró diferencias significativas entre meses en 2005 (Kruskal Wallis- $\mathrm{H}=17.9, \mathrm{p}<0.0001$ ), 2006 (Kruskal Wallis-H $=19.0, \mathrm{p}<0.001$ ) y 2007 2008 (Kruskal Wallis-H $=20.6, \mathrm{p}<0.0001$ ). 

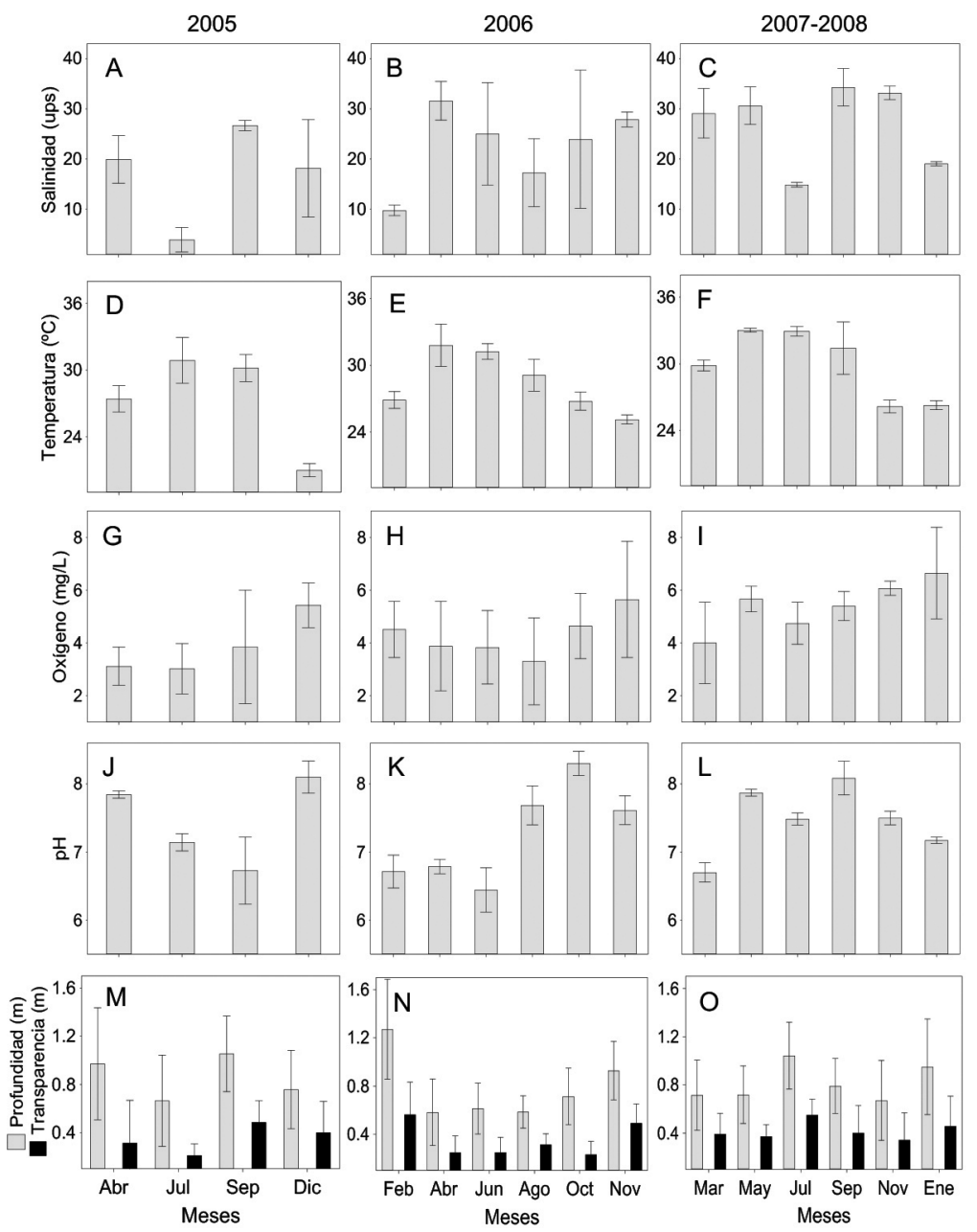

Fig. 3. Variación temporal de los parámetros físicoquímicos en el sistema lagunar La Mancha.

Fig. 3. Temporal variation of the physicochemical parameters in the La Mancha Lagoon System.

La temperatura del agua registró el valor más bajo $\left(20.9{ }^{\circ} \mathrm{C} \pm 0.6\right)$ en diciembre 2005 y el más alto $\left(33.0^{\circ} \mathrm{C} \pm 0.2\right)$ en mayo 2007 (Fig. 3D a F). Este parámetro indicó diferencias significativas entre meses en 2005 (Kruskal Wallis-H $=21.9, \mathrm{p}<0.0001), 2006$ (Kruskal Wallis- $\mathrm{H}=$ $35.5, \mathrm{p}<0.0001)$ y $2007-2008$ (Kruskal Wallis$\mathrm{H}=25.9, \mathrm{p}<0.0001)$. El oxígeno disuelto mostró un comportamiento inverso a la temperatura con el valor promedio más bajo (3.02 $\mathrm{mg} / \mathrm{L} \pm 0.95)$ en julio 2005 y el más alto $(6.6$ $\mathrm{mg} / \mathrm{L} \pm 1.8$ ) en enero 2008 (Fig. 3G a I). Se presentaron diferencias significativas en 2005 (Kruskal Wallis- $\mathrm{H}=9.8, \mathrm{p}<0.05$ ) y 2007-2008
(Kruskal Wallis- $\mathrm{H}=23.7, \mathrm{p}<0.0001$ ). El valor promedio de $\mathrm{pH}$ más bajo se registró en junio $2006(6.4 \pm 0.3)$, mientras que valores más alcalinos en octubre $2006(8.3 \pm 0.2)$ (Fig. 3J a L). Este parámetro mostró diferencias significativas en 2005 (Kruskal Wallis- $\mathrm{H}=22.9, \mathrm{p}<$ 0.0001), 2006 (ANOVA-F $=0.05, \mathrm{p}<0.0001$ ) y 2007 (Kruskal Wallis- $\mathrm{H}=38.1, \mathrm{p}<0.00001$ ). Cambios en los niveles de agua se observaron en los meses de estudio con profundidades mínimas promedio $(0.6 \mathrm{~m} \pm 0.3)$ en abril 2006 y máximas promedio $(1.3 \mathrm{~m} \pm 0.4)$ en febrero 2006 (Fig. 3M a O). La transparencia presentó el valor promedio más bajo $(0.21 \mathrm{~m} \pm 0.09)$ 
en julio 2005, mientras que el valor promedio más alto $(0.56 \mathrm{~m} \pm 0.26)$ en febrero 2006 (Fig. $3 \mathrm{M}$ a O). Sólo se presentaron diferencias significativas entre meses del 2006 para valores promedio de profundidad (ANOVA-F $=0.07$, $\mathrm{p}<0.001)$ y transparencia (ANOVA-F $=0.02$, $\mathrm{p}<0.05)$. El Cuadro 1 muestra los valores promedios anuales de los parámetros físicoquímicos en el sistema.
Composición ictiofaunística: En el sistema lagunar La Mancha, se recolectaron un total de 5974 peces pertenecientes a 11 órdenes, 25 familias, 39 géneros y 50 especies (Cuadro 2). Las familias que presentaron mayor número de especies fueron Gerreidae con siete especies: Diapterus auratus, D. rhombeus, Eucinostomus argenteus, E. gula, E. melanopterus, Eugerres plumieri y Gerres cinereus; Engraulidae

CUADRO 1

Valores promedio y estimadores estadísticos de los parámetros físicoquímicos para los años de muestreo en el sistema lagunar La Mancha, Veracruz

TABLE 1

Average values and statistical estimators of the physicochemical parameters for the sampling years in the La Mancha Lagoon System, Veracruz

\begin{tabular}{lccccc}
\multicolumn{1}{c}{ Parámetro } & 2005 & 2006 & $2007-2008$ & Estimador & $p$ \\
Salinidad (ups) & $17.18 \pm 9.9$ & $22.58 \pm 10.3$ & $26.86 \pm 7.90$ & $\mathrm{H}=16.54$ & $0.0003^{*}$ \\
Temperatura $\left({ }^{\circ} \mathrm{C}\right)$ & $27.37 \pm 4.2$ & $28.50 \pm 2.70$ & $29.94 \pm 3.04$ & $\mathrm{H}=7.08$ & $0.030^{*}$ \\
Oxígeno disuelto $(\mathrm{mg} / \mathrm{L})$ & $3.85 \pm 1.57$ & $4.30 \pm 1.67$ & $5.42 \pm 1.32$ & $\mathrm{~F}=10.30$ & $<0.0001^{*}$ \\
$\mathrm{pH}$ & $7.45 \pm 0.62$ & $7.26 \pm 0.70$ & $7.47 \pm 0.48$ & $\mathrm{H}=2.67$ & 0.26 \\
Profundidad $(\mathrm{m})$ & $0.86 \pm 0.39$ & $0.78 \pm 0.35$ & $0.81 \pm 0.31$ & $\mathrm{~F}=0.43$ & 0.65 \\
Transparencia $(\mathrm{m})$ & $0.35 \pm 0.25$ & $0.35 \pm 0.20$ & $0.42 \pm 0.19$ & $\mathrm{~F}=1.37$ & 0.26 \\
\hline
\end{tabular}

*Parámetros con diferencias significativas

CUADRO 2

Listado de las especies de peces colectadas en el sistema lagunar La Mancha, Veracruz

TABLE 2

List of the fish species collected in the La Mancha Lagoon System, Veracruz

\begin{tabular}{llccccccc}
\multicolumn{1}{c}{ Familia } & \multicolumn{1}{c}{ Especies } & $\mathrm{AB}$ & Número (\%) & Peso (\%) & Frecuencia (\%) & IVI $(\%)$ & $\mathrm{CC}$ & $\mathrm{CT}$ \\
Dasyatidae & Hypanus sabinus & $\mathrm{Hs}$ & 0.05 & 5.93 & 0.48 & 6.46 & $\mathrm{ME}$ & $3^{\circ}$ \\
Elopidae & Elops saurus & $\mathrm{Es}$ & 0.07 & 0.50 & 0.64 & 1.20 & $\mathrm{ME}$ & $3^{\circ}$ \\
Megalopidae & Megalops atlanticus & $\mathrm{Ma}$ & 0.02 & 0.77 & 0.16 & 0.95 & $\mathrm{ME}$ & $3^{\circ}$ \\
Clupeidae & Brevoortia gunteri & $\mathrm{Bg}$ & 0.03 & 0.01 & 0.16 & 0.19 & $\mathrm{ME}$ & $1^{\circ}$ \\
Clupeidae & Harengula jaguana & $\mathrm{Hj}$ & 0.10 & 0.04 & 0.16 & 0.30 & $\mathrm{ME}$ & $1^{\circ}$ \\
Engraulidae & Cetengraulis edentulus & $\mathrm{Ce}$ & 0.30 & 0.03 & 0.32 & 0.65 & $\mathrm{ME}$ & $1^{\circ}$ \\
Engraulidae & Anchovia clupeoides & $\mathrm{Ac}$ & 0.13 & 0.02 & 0.48 & 0.63 & $\mathrm{ME}$ & $1^{\circ}$ \\
Engraulidae & Anchoa hepsetus & $\mathrm{Ah}$ & 0.15 & 0.03 & 0.32 & 0.49 & $\mathrm{ME}$ & $1^{\circ}$ \\
Engraulidae & Anchoa lamprotaenia & $\mathrm{Ala}$ & 0.28 & 0.06 & 0.32 & 0.66 & $\mathrm{ME}$ & $1^{\circ}$ \\
Engraulidae & Anchoa mitchilli & $\mathrm{Am}$ & 30.20 & 4.20 & 9.38 & 43.75 & $\mathrm{ME}$ & $1^{\circ}$ \\
Ariidae & Sciades felis & $\mathrm{Sf}$ & 0.08 & 2.30 & 0.64 & 2.98 & $\mathrm{ME}$ & $3^{\circ}$ \\
Ariidae & Cathorops aguadulce & $\mathrm{Ca}$ & 17.60 & 33.0 & 10.49 & 61.07 & $\mathrm{E}$ & $2^{\circ}$ \\
Batrachoididae & Opsanus beta & $\mathrm{Ob}$ & 0.05 & 0.21 & 0.48 & 0.74 & $\mathrm{ME}$ & $3^{\circ}$ \\
Mugilidae & Mugil curema & $\mathrm{Mc}$ & 2.20 & 9.10 & 4.93 & 16.17 & $\mathrm{ME}$ & $1^{\circ}$ \\
Atherinidae & Atherinomorus stipes & $\mathrm{Ast}$ & 0.02 & 0.01 & 0.16 & 0.18 & $\mathrm{ME}$ & $1^{\circ}$ \\
\hline
\end{tabular}


CUADRO 2 (Continuación) / TABLE 2 (Continued)

\begin{tabular}{|c|c|c|c|c|c|c|c|c|}
\hline Familia & Especies & $\mathrm{AB}$ & Número (\%) & Peso $(\%)$ & Frecuencia $(\%)$ & IVI (\%) & $\mathrm{CC}$ & $\mathrm{CT}$ \\
\hline Hemirhamphidae & Hemirhamphus brasiliensis & $\mathrm{Hb}$ & 0.30 & 0.53 & 0.64 & 1.45 & ES & $1^{\circ}$ \\
\hline Hemirhamphidae & Hyporhamphus meeki & $\mathrm{Hm}$ & 0.02 & 0.07 & 0.16 & 0.24 & ME & $1^{\circ}$ \\
\hline Belonidae & Strongylura timucu & St & 0.03 & 0.08 & 0.32 & 0.43 & ES & $3^{\circ}$ \\
\hline Belonidae & Strongylura notata & $\mathrm{Sn}$ & 2.80 & 9.20 & 2.23 & 14.20 & $\mathrm{ME}$ & $3^{\circ}$ \\
\hline Synbranchidae & Ophisternon aenigmaticum & $\mathrm{Oa}$ & 0.02 & 0.02 & 0.16 & 0.19 & $\mathrm{D}$ & $3^{\circ}$ \\
\hline Centropomidae & Centropomus undecimalis & Cun & 0.30 & 1.60 & 2.38 & 4.27 & $\mathrm{ME}$ & $3^{\circ}$ \\
\hline Centropomidae & Centropomus pectinatus & Cpe & 0.08 & 0.40 & 0.48 & 0.96 & $\mathrm{ME}$ & $2^{\circ}$ \\
\hline Centropomidae & Centropomus parallelus & $\mathrm{Cp}$ & 2.66 & 3.13 & 6.52 & 12.31 & $\mathrm{ME}$ & $3^{\circ}$ \\
\hline Carangidae & Oligoplites saurus & Os & 0.20 & 0.04 & 1.43 & 1.66 & $\mathrm{ME}$ & $3^{\circ}$ \\
\hline Carangidae & Trachinotus falcatus & $\mathrm{Tf}$ & 0.03 & 0.01 & 0.32 & 0.35 & ES & $3^{\circ}$ \\
\hline Carangidae & Selene vomer & $\mathrm{Sv}$ & 0.08 & 0.27 & 0.79 & 1.14 & ES & $3^{\circ}$ \\
\hline Carangidae & Hemicaranx amblyrhynchus & На & 1.41 & 0.70 & 2.23 & 4.30 & ES & $3^{\circ}$ \\
\hline Lutjanidae & Lutjanus cyanopterus & $\mathrm{Lc}$ & 0.02 & 0.02 & 0.16 & 0.19 & $\mathrm{ME}$ & $3^{\circ}$ \\
\hline Lutjanidae & Lutjanus griseus & $\operatorname{Lg}$ & 0.52 & 1.40 & 1.59 & 3.49 & $\mathrm{ME}$ & $3^{\circ}$ \\
\hline Gerreidae & Gerres cinereus & $\mathrm{Gc}$ & 0.03 & 0.14 & 0.16 & 0.33 & ES & $1^{\circ}$ \\
\hline Gerreidae & Eucinostomus melanopterus & Em & 3.98 & 0.64 & 3.97 & 8.60 & $\mathrm{ME}$ & $1^{\circ}$ \\
\hline Gerreidae & Eucinostomus gula & $\mathrm{Eg}$ & 0.17 & 0.03 & 0.64 & 0.83 & $\mathrm{ME}$ & $1^{\circ}$ \\
\hline Gerreidae & Eucinostomus argenteus & $\mathrm{Ea}$ & 1.54 & 0.02 & 0.79 & 2.36 & $\mathrm{ME}$ & $1^{\circ}$ \\
\hline Gerreidae & Diapterus rhombeus & $\operatorname{Dr}$ & 18.50 & 6.22 & 10.17 & 34.88 & $\mathrm{ME}$ & $1^{\circ}$ \\
\hline Gerreidae & Diapterus auratus & $\mathrm{Da}$ & 8.94 & 2.80 & 9.06 & 20.80 & $\mathrm{ME}$ & $1^{\circ}$ \\
\hline Gerreidae & Eugerres plumieri & Ep & 1.24 & 4.31 & 4.29 & 9.84 & $\mathrm{ME}$ & $1^{\circ}$ \\
\hline Haemulidae & Anisotremus surinamensis & As & 0.02 & 0.07 & 0.32 & 0.40 & $\mathrm{ME}$ & $2^{\circ}$ \\
\hline Haemulidae & Pomadasys crocro & Pc & 0.02 & 0.02 & 0.16 & 0.19 & ME & $3^{\circ}$ \\
\hline Sparidae & Archosargus probatocephalus & Ap & 0.08 & 1.32 & 0.32 & 1.72 & $\mathrm{ME}$ & $2^{\circ}$ \\
\hline Sciaenidae & Micropogonias undulatus & $\mathrm{Mu}$ & 0.13 & 0.25 & 0.16 & 0.54 & $\mathrm{ME}$ & $3^{\circ}$ \\
\hline Sciaenidae & Bairdiella chrysoura & $\mathrm{Bc}$ & 0.47 & 1.08 & 2.07 & 3.61 & $\mathrm{ME}$ & $2^{\circ}$ \\
\hline Sciaenidae & Bairdiella ronchus & $\mathrm{Br}$ & 2.61 & 7.40 & 6.84 & 16.84 & $\mathrm{ME}$ & $2^{\circ}$ \\
\hline Cichlidae & Mayaheros urophtalmus & Mur & 0.02 & 0.19 & 0.16 & 0.37 & $\mathrm{D}$ & $1^{\circ}$ \\
\hline Eleotridae & Gobiomorus dormitor & $\mathrm{Gd}$ & 0.10 & 0.60 & 0.64 & 1.34 & $\mathrm{E}$ & $3^{\circ}$ \\
\hline Gobiidae & Bathygobius soporator & Bs & 0.07 & 0.01 & 0.16 & 0.24 & $\mathrm{ME}$ & $3^{\circ}$ \\
\hline Gobiidae & Gobionellus oceanicus & Go & 0.89 & 0.32 & 4.13 & 5.34 & ES & $2^{\circ}$ \\
\hline Sphyraenidae & Sphyraena barracuda & $\mathrm{Sb}$ & 0.07 & 0.42 & 0.32 & 0.81 & ES & $3^{\circ}$ \\
\hline Paralichthyidae & Citharichthys spilopterus & $\mathrm{Cs}$ & 1.21 & 0.62 & 4.93 & 6.76 & $\mathrm{ME}$ & $3^{\circ}$ \\
\hline Paralichthyidae & Citharichthys uhleri & Cuh & 0.02 & 0.01 & 0.16 & 0.18 & $\mathrm{ME}$ & $3^{\circ}$ \\
\hline Achiridae & Achirus lineatus & Ali & 0.30 & 0.04 & 2.07 & 2.41 & $\mathrm{ME}$ & $2^{\circ}$ \\
\hline
\end{tabular}

Abreviaturas (AB) utilizadas en la figura 8 (Análisis de Correspondencia Canónica). Índice de Valor de Importancia (IVI): - especies dominantes. Componente comunitario $(\mathrm{CC})$ : $\mathrm{ME}=$ marino eurihalino, $\mathrm{ES}=$ marino estenohalino, $\mathrm{E}=\mathrm{estuarino}$, $\mathrm{D}=$ dulceacuícola. Categoría trófica $(\mathrm{CT}): 1^{\circ}=$ primer orden, $2^{\circ}=$ segundo orden, $3^{\circ}=$ tercer orden.

Abbreviations $(\mathrm{AB})$ used in figure 8 are shown (Canonical Correspondence Analysis). Importance Value Index (IVI): - dominant species. Community inhabitant $(\mathrm{CC})$ : $\mathrm{ME}=$ marine euryhaline, $\mathrm{ES}=$ marine estenohaline, $\mathrm{E}=$ estuarine, $\mathrm{D}=$ freshwater. Trophic category $(\mathrm{CT}): 1^{\circ}=$ first order, $2^{\circ}=$ second order, $3^{\circ}=$ third order.

con cinco especies: Anchoa hepsetus, A. lamprotaenia, A. mitchilli, Anchovia clupeoides y Cetengraulis edentulus; Carangidae con cuatro especies: Hemicaranx amblyrhynchus, Oligoplites saurus, Selene vomer y Trachinotus falcatus; Centropomidae con tres especies:
Centropomus parallelus, $C$. pectinatus y $C$. undecimalis; y Scianidae con tres especies: Bairdiella chrysoura, B. ronchus y Micropogonias undulatus. El número de especies varió de $37(74 \%)$ en 2005, 33 (66\%) en 2006 y 32 (64\%) en 2007-2008. Cinco especies resultaron 

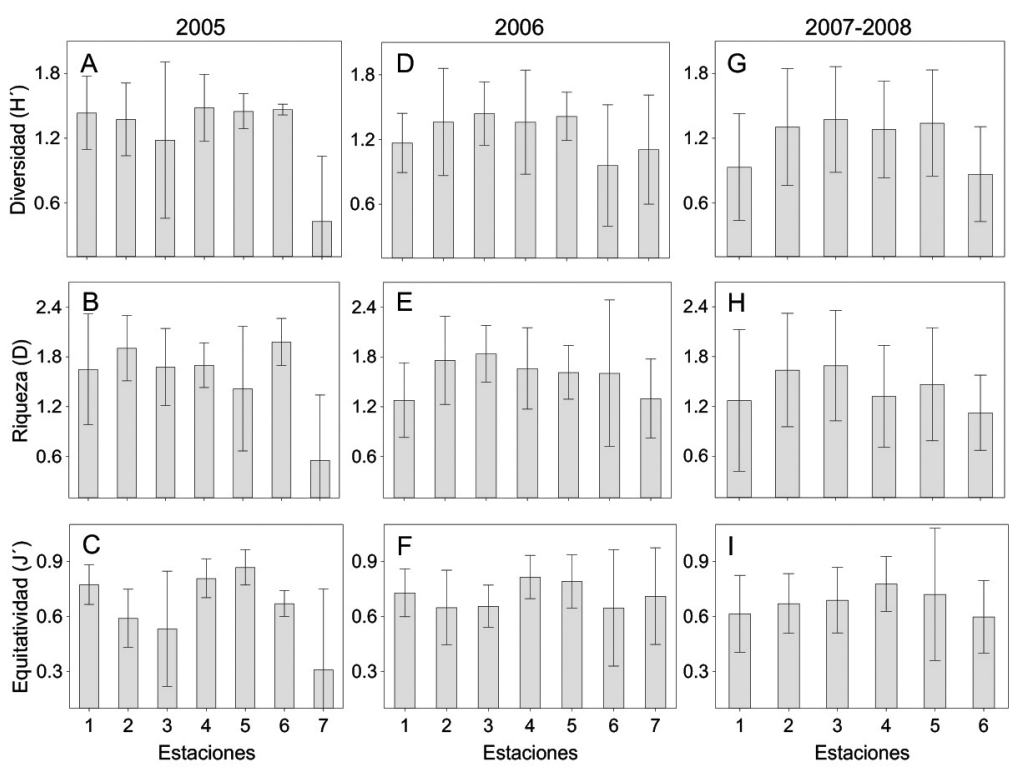

Fig. 4. Variación espacial de los índices de diversidad H', D y J' de la comunidad de peces en el sistema lagunar La Mancha. Fig. 4. Spatial variation of the diversity indices H', D and J' for the fish community in the La Mancha Lagoon System.

registros nuevos para el sistema lagunar $\mathrm{La}$ Mancha: Brevoortia gunteri (2005), M. undulatus (2005), A. lamprotaenia (2006), Atherinomorus stipes (2007) y O. aenigmaticum (2007).

Parámetros ecológicos de la comunidadescala espacial: Comparando los parámetros de diversidad (H'), riqueza (D) y equitatividad (J') para el 2005, los valores promedio más altos de $\mathrm{H}^{\prime}(1.48 \pm 0.22)$ se registraron en la estación 4 , D $(1.98 \pm 0.20)$ en la estación 6 y $\mathrm{J}^{\prime}(0.87 \pm 0.09)$ en la estación $5, \mathrm{y}$ los más bajos $\left(\mathrm{H}^{\prime}=0.43 \pm 0.61 ; \mathrm{D}=0.56 \pm 0.78\right.$ y J'=0.31 \pm 0.43 ) en la estación 7 (Fig. 4A a C). Durante 2006 y 2007-2008, se observó un comportamiento similar de los índices, con los valores promedio más altos en la estación $3\left(\mathrm{H}^{\prime}=1.44\right.$ \pm 0.3 y $1.38 \pm 0.49 ; \mathrm{D}=1.84 \pm 0.34$ y 1.69 $\pm 0.67)$ y J' $(0.81 \pm 0.65$ y $0.78 \pm 0.15)$ en la estación 4 (Fig. 4D a I). El análisis de varianza mostró diferencias significativas entre sitios sólo para $\mathrm{H}^{\prime}$ en 2005 (Kruskal Wallis- $\mathrm{H}=5.17$, $\mathrm{p}<0.0001)$.

Parámetros ecológicos de la comunidadescala temporal: Entre los meses de muestreo, los valores promedio más altos de diversidad $\left(\mathrm{H}^{\prime}=1.52 \pm 0.23\right)$ se presentaron en septiembre 2005 (Fig. 5A), D $(1.99 \pm 0.46)$ en noviembre y $\mathrm{J}^{\prime}(0.87 \pm 0.05)$ en marzo, ambos en 2007 (Fig. 5H y 5I). Por otra parte, los promedios más bajos para los parámetros de diversidad y equitatividad $\left(\mathrm{H}^{\prime}=0.59 \pm 0.67\right.$ y $\mathrm{J}^{\prime}=0.28 \pm$ 0.29 ) se presentaron en febrero 2006 (Fig. 5D y F) y para la riqueza $(\mathrm{D}=0.77 \pm 0.3)$ en julio 2007 (Fig. 5H). Se encontraron diferencias significativas entre meses durante 2006 para H' (ANOVA-F $=0.20, \mathrm{p}<0.05)$ y J' (Kruskal Wallis- $\mathrm{H}=14.21, \mathrm{p}<0.05)$; para 2007-2008 en diversidad (ANOVA-F $=3.0, \mathrm{p}<0.05$ ) y riqueza de especies (ANOVA-F $=3.1, \mathrm{p}<0.05$ ). El Cuadro 3 presenta los valores promedios entre años de estos parámetros, no se presentaron diferencias significativas $(\mathrm{p}>0.05)$.

Abundancia-escala espacial: Entre sitios de muestreo, los valores promedio más altos de densidad (0.62 ind. $\left./ \mathrm{m}^{2} \pm 0.68\right)$ y biomasa $(3.43$ $\left.\mathrm{g} / \mathrm{m}^{2} \pm 3.01\right)$ se presentaron en la estación 2 del 2005 (Fig. 6A y B), y el peso promedio (29.6 g/ind. \pm 22.10$)$ en la estación 5 del 2007-2008 (Fig. 6I). Los promedios más bajos de densidad 
CUADRO 3

Valores promedio y estimadores estadísticos de los índices de diversidad y abundancia calculados para los años de muestreo en el sistema lagunar La Mancha, Veracruz

TABLE 3

Average values and statistical estimators of diversity and abundance indexes calculated for the sampling years in the La Mancha Lagoon System, Veracruz

\begin{tabular}{lccccc}
\multicolumn{1}{c}{ Parámetro } & 2005 & 2006 & $2007-2008$ & Estimador & $p$ \\
Diversidad (H') & $1.30 \pm 0.48$ & $1.24 \pm 0.49$ & $1.19 \pm 0.49$ & $\mathrm{~F}=0.32$ & 0.73 \\
Riqueza (D) & $1.61 \pm 0.59$ & $1.51 \pm 0.62$ & $1.43 \pm 0.65$ & $\mathrm{~F}=0.56$ & 0.57 \\
Equitatividad (J') & $0.66 \pm 0.24$ & $0.69 \pm 0.23$ & $0.68 \pm 0.21$ & $\mathrm{~F}=0.09$ & 0.91 \\
Densidad (ind./m ${ }^{2}$ ) & $0.32 \pm 0.43$ & $0.11 \pm 0.08$ & $0.09 \pm 0.06$ & $\mathrm{H}=5.63$ & 0.06 \\
Biomasa (g/m ${ }^{2}$ ) & $1.92 \pm 2.04$ & $1.15 \pm 1.85$ & $1.32 \pm 1.18$ & $\mathrm{~F}=1.54$ & 0.22 \\
Peso promedio (g/ind.) & $11.81 \pm 9.87$ & $12.99 \pm 14.06$ & $18.56 \pm 15.84$ & $\mathrm{~F}=2.11$ & 0.13 \\
\hline
\end{tabular}
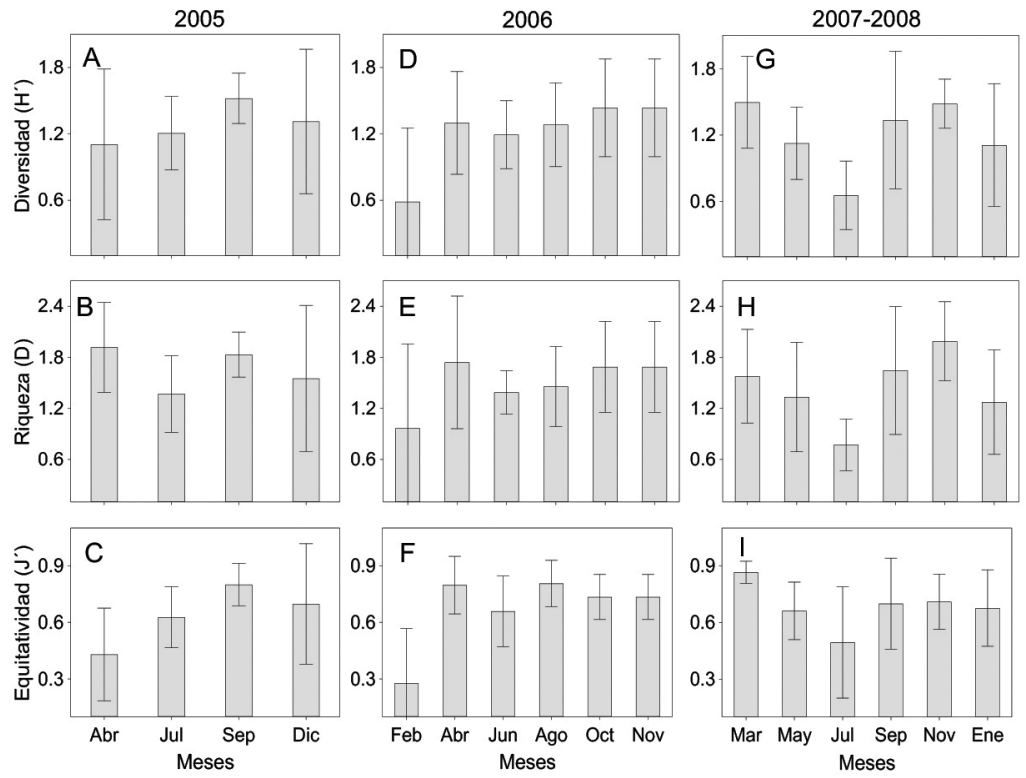

Fig. 5. Variación temporal de los índices de diversidad H', D y J' de la comunidad de peces en el sistema lagunar La Mancha. Fig. 5. Temporal variation of the diversity indices H', D and J' for the fish community in the La Mancha Lagoon System.

(0.02 ind. $\left./ \mathrm{m}^{2} \pm 0.04\right)$, biomasa $\left(0.12 \mathrm{~g} / \mathrm{m}^{2} \pm\right.$ $0.15)$ y peso promedio $(5.10 \mathrm{~g} /$ ind. \pm 1.13$) \mathrm{se}$ registraron en la estación 7 del 2005 (Fig. 6A a C). Las especies con mayor densidad fueron A. mitchilli y D. rhombeus (estaciones 2 y 3), mientras que la mayor biomasa la aportó Hypanus sabinus, Strongylura notata, Mugil curema (estación 1 y 2), y Cathorops aguadulce (estación 6 y 7). El análisis de varianza para densidad (ANOVA-F $=0.003, \mathrm{p}<0.001)$, biomasa (Kruskal Wallis- $\mathrm{H}=8.20, \mathrm{p}<0.05$ ) y peso promedio (Kruskal Wallis- $\mathrm{H}=3.09, \mathrm{p}<$ $0.05)$ indicaron diferencias significativas entre sitios sólo en 2006.

Abundancia-escala temporal: Entre meses de muestreo se observó que los valores promedio más altos de densidad (1.17 ind./ $\left.\mathrm{m}^{2} \pm 0.60\right)$ y biomasa $\left(4.33 \mathrm{~g} / \mathrm{m}^{2} \pm 2.58\right) \mathrm{se}$ presentaron en abril del 2005 (Figs. 7A y 7B), 


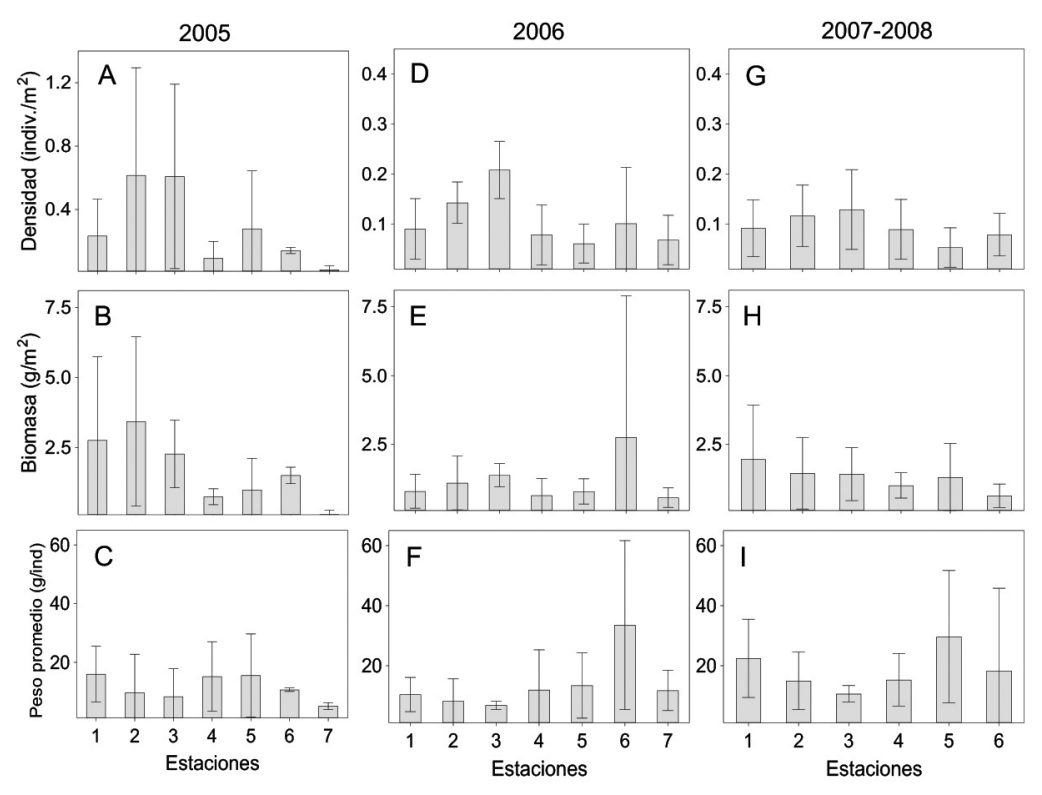

Fig. 6. Variación espacial de la densidad, biomasa y peso promedio de la comunidad de peces en el sistema lagunar La Mancha.

Fig. 6. Spatial variation in density, biomass and average weight for the fish community in the La Mancha Lagoon System.

y el peso promedio $(30.82 \mathrm{~g} / \mathrm{ind} . \pm 23.1)$ en julio del 2007 (Fig. 7I). Los valores promedio más bajos de densidad $\left(0.06\right.$ ind. $\left./ \mathrm{m}^{2} \pm 0.05\right)$ se registraron en julio de 2007, de biomasa (0.68 $\left.\mathrm{g} / \mathrm{m}^{2} \pm 0.30\right)$ en agosto de 2006 y peso promedio (3.85 g/ind. \pm 1.13 ) en abril de 2005 (Figs. 7A a 7I). Durante abril (2005) las especies $A$. mitchilli y $D$. rhombeus fueron las más abundantes en número, mientras que $C$. aguadulce (junio 2006), H. sabinus (noviembre 2007) y $S$. notata (septiembre 2007) presentaron la mayor biomasa. Durante julio y enero se registraron las tallas más grandes de especies como $B$. ronchus (190-295 mm LT), C. aguadulce (140$272 \mathrm{~mm} \mathrm{LT})$, C. parallelus (120-255 mm LT), D. auratus (105-152 mm LT) y S. notata (192$450 \mathrm{~mm} \mathrm{LT}$ ) que corresponden a organismos preadultos y adultos, mientras las tallas jóvenes $(<150 \mathrm{~mm} \mathrm{LT})$ se registraron en abril-junio (secas). El análisis de varianza entre meses indicó diferencias significativas para densidad (Kruskal Wallis-H $=12.35, \mathrm{p}<0.001$ ) en 2005, para biomasa (Kruskal Wallis-H $=9.68, \mathrm{p}<$ 0.05 ) en 2006, y para peso promedio (Kruskal Wallis-H $=12.83, \mathrm{p}<0.05)$ en 2007-2008. Los valores más altos de abundancia promedio anuales se muestran en el cuadro 3 , el análisis de varianza no mostró diferencias significativas entre años ( $p>0.05)$.

El índice del valor de importancia (IVI) indicó que cuatro especies fueron dominantes para el sistema: C. aguadulce, A. mitchilli, D. rhombeus y $D$. auratus, las cuales representaron el $75 \%$ en número y el $46 \%$ en peso de la captura total. La especie C. aguadulce presentó el valor más alto de IVI con los mayores porcentajes en peso (Cuadro 2). De acuerdo con la clasificación de componentes comunitarios, 37 (74\%) especies fueron marinas eurihalinas, 9 (18\%) fueron estenohalinas, 2 (4\%) estuarinas y $2(4 \%)$ dulceacuícolas. Por su alimentación y hábitos alimentarios, 23 (46\%) especies son consumidoras de tercer orden, 19 (38\%) son de primer orden y $8(16 \%)$ de segundo orden. 

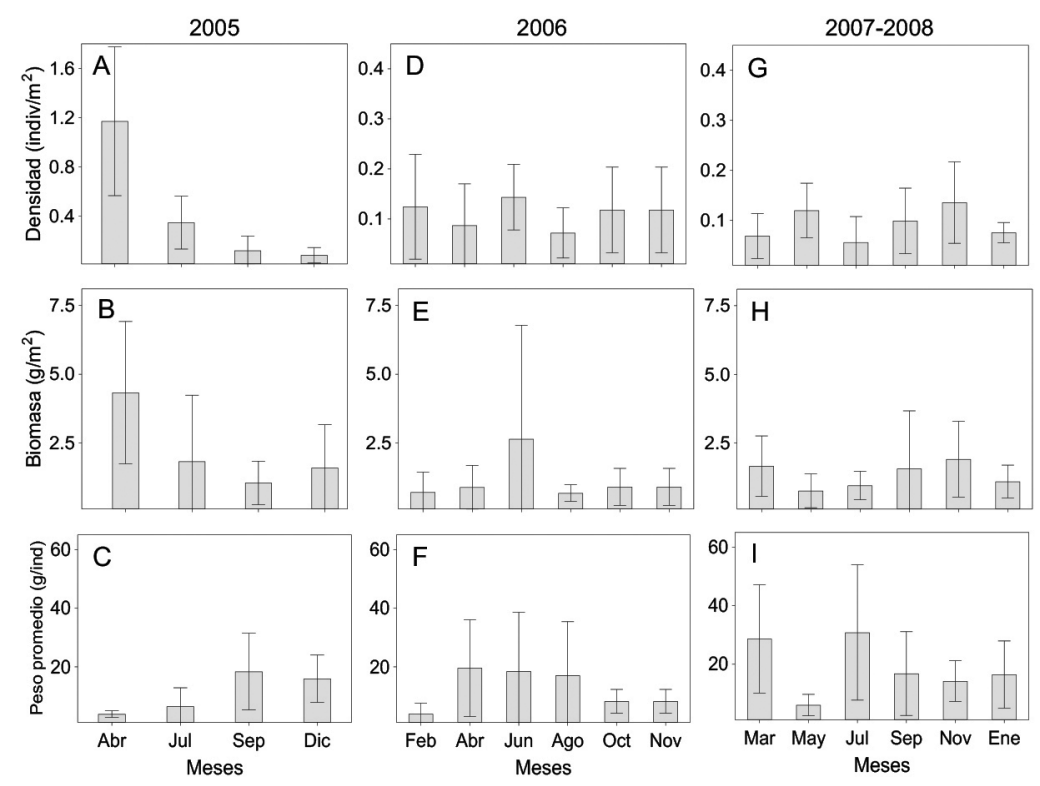

Fig. 7. Variación temporal de la densidad, biomasa y peso promedio de la comunidad de peces en el sistema lagunar La Mancha.

Fig. 7. Temporal variation in density, biomass and average weight for the fish community in the La Mancha Lagoon System.

Se observó que especies carnívoras de tercer orden se distribuyeron principalmente en la zona norte-este del sistema (e.g. C. parallelus, $H$. amblyrhynchus), donde son abundantes los crustáceos en áreas de pastos marinos, mientras que la mayor abundancia de consumidores de primer orden (e.g. A. clupeoides, E. melanopterus) se registraron en la parte central y sur de la laguna.

Análisis peces-hábitat: $\mathrm{El}$ análisis de correspondencia canónica (ACC) mostró que la relación entre la abundancia de las especies, los sitios de muestreo y las variables ambientales fue explicada en un $57 \%$ de la varianza total para los dos ejes de ordenación, con eigenvalores de 0.29 (eje 1) y 0.16 (eje 2). El ACC explicó el $36 \%$ de la variación especies-ambiente para el eje 1 , señalando que los factores que determinan en mayor medida la composición y distribución de las especies son la temperatura $(\mathrm{r}=0.93)$, salinidad $(\mathrm{r}=$ $0.56)$ y oxígeno disuelto $(\mathrm{r}=0.55)$ (Fig. 8). Las especies que mostraron mayor correlación con el eje 1 fueron A. surinamensis, A. probatocephalus, Bathygobius soporator, C. edentulus, G. cinereus, H. brasiliensis, H. meeki, Lutjanus griseus, Pomadasys crocro, Selene vomer, $S$. notata, S. barracuda y T. falcatus de los componentes eurihalino y estenohalino (Cuadro 2). Estas especies presentaron abundancias de bajas a intermedias, asociadas con zonas someras (estaciones 1 y 2 ) de mayor salinidad y transparencia $(\mathrm{r}=-0.34)$. El eje 2 explicó el $21 \%$ de la variación especies-ambiente, donde el oxígeno disuelto $(\mathrm{r}=0.45)$, la salinidad $(\mathrm{r}=$ $0.40)$ y la profundidad $(\mathrm{r}=-0.83)$ son los factores más importantes en la distribución de las especies a lo largo de este eje. Las especies que mostraron mayor correlación con el eje 2 fueron $A$. hepsetus, $A$. clupeoides, $A$. stipes, $B$. ronchus, C. aguadulce, D. rhombeus, E. plumieri, E. saurus, Harengula jaguana, L. cyanopterus, M. undulatus, Citharichthys uhleri, M. atlanticus, M. urophtalmus, $S$. felis y $O$. aenigmaticum que pertenecen a los componentes eurihalino 


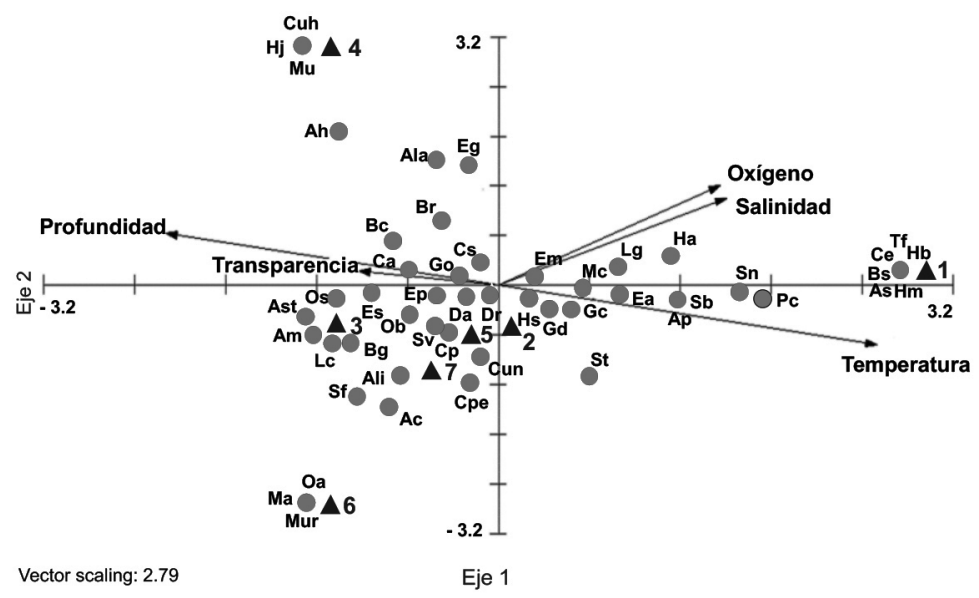

Fig. 8. Diagrama del Análisis de Correspondencia Canónica de la abundancia de las especies, las variables ambientales y los sitios de muestreo en el sistema lagunar La Mancha. La longitud y dirección de las flechas indican la importancia relativa de las variables ambientales. Las abreviaturas de las especies (AB) se indican en Cuadro 2.

Fig. 8. Canonical Correspondence Analysis Plot of species abundance, environmental variables and the sampling sites in the La Mancha Lagoon System. Arrow length and direction indicate the relative importance of environmental variables. Species abbreviations $(\mathrm{AB})$ are listed in table 2 .

y dulceacuícola (Cuadro 2). Estas especies presentaron abundancias bajas y altas, asociadas a zonas de mayor profundidad con menor salinidad y transparencia (estaciones 3, 5, 6 y 7).

\section{DISCUSIÓN}

En el sistema lagunar La Mancha, la variabilidad de los parámetros físicoquímicos se relacionó principalmente con la mayor descarga de los ríos en la porción sur, así como por la entrada de agua marina en el norte, ambos procesos determinados por los cambios estacionales en las distintas épocas climáticas de la región (Díaz-Ruiz et al., 2008). El sistema presentó un gradiente salino que varió desde condiciones marinas en el extremo norte de la laguna (estaciones 1 a 4), a condiciones mesohalinas con los menores valores en la parte sur (estaciones 5 a 7 ) debido al aporte del Río Caño Grande y Río Sábalo. La variación temporal de la salinidad dentro de la laguna estuvo relacionada con una alta precipitación y máxima descarga de los ríos durante los meses de la época de lluvias como ocurrió en julio del 2005 y 2007, respectivamente, donde se registraron las salinidades más bajas. Mientras que las salinidades más altas se registraron en abril 2006 y septiembre del 2007, como resultado de la poca o nula precipitación, y al incremento en la insolación y evaporación que ocurrió durante esos meses (Lara-Domínguez et al., 2006). Durante los meses de nortes la salinidad fue menor (febrero 2006) por el efecto de dilución causado por las lluvias de la temporada previa. La temperatura del agua mantuvo un comportamiento similar entre los sitios evaluados, y mostró poca variación desde la boca hacia el interior de la laguna, debido a que el sistema es somero y con buena mezcla vertical (Snedden et al., 2013). La temperatura anual se relacionó con las temporadas climáticas, con la mayor temperatura en los meses más cálidos (abril-mayo-julio) del periodo primavera-verano y la menor durante los meses más fríos (noviembre-diciembre) del periodo otoño-invierno. Los niveles más altos de oxígeno en la columna de agua se presentaron en la zona norte de la laguna, debido a los procesos de mezcla y corrientes que ocurren en las cercanías de la boca con el mar (Lara-Domínguez et al., 2006). El oxígeno disuelto también presentó un patrón relacionado a la estacionalidad 
climática, con mayor concentración durante los meses de noviembre y diciembre de 2005 y 2007, relacionado con las bajas temperaturas y acción del viento de la temporada de nortes y la menor durante la época de lluvias, atribuida al incremento de materia orgánica por la descarga de los ríos en la parte sur del sistema (De la Lanza Espino, Rodríguez, Baus, \& Pulido, 2011; Aguirre-León et al., 2014). Respecto a los valores de $\mathrm{pH}$, estos se comportaron dentro del intervalo normal de 6.5 a 8.5 los cuales están dentro de la variación encontrada en otras lagunas costeras similares (De la Lanza Espino et al., 2011). Los valores de $\mathrm{pH}$ fueron mayores en el periodo otoño-invierno (2005 y 2006), mientras que los más bajos en el periodo primavera-verano (2005-2007). Las variaciones de $\mathrm{pH}$ estuvieron asociadas al intercambio de agua de mar, a la lluvia, cantidad de escurrimientos, actividad biológica por organismos, procesos biogeoquímicos, degradación de materia orgánica o la remoción de sedimentos por corrientes causando remineralización (López-Ortega et al., 2010; TorresAlvarado, Calva-Benítez, Álvarez-Hernández, \& Trejo-Aguilar, 2016). La profundidad y transparencia mostraron un comportamiento inverso entre las localidades de muestreo, con la menor profundidad en la boca de conexión, debido a los procesos de azolve que existe en esta zona, mientras que la menor transparencia, se relacionó con las descargas de los ríos en la parte sur del sistema. Durante los meses de lluvias de 2005 y 2007, se registró la mayor profundidad debido al incremento del volumen de agua por los aportes fluviales y las precipitaciones en el sistema. Un comportamiento similar de estos parámetros fue observado en ecosistemas parecidos por Aguirre-León, DíazRuiz y Ramírez-Huerta, (2010), Díaz-Ruiz et al. (2012), Sosa-López et al., (2012) quienes indicaron que gran parte de los procesos biológicos y ecológicos de las especies de peces, son afectados por las variaciones físicoquímicas del agua en estos sistemas.

En este trabajo se identificaron 50 especies de peces entre 2005 y 2008. En otros estudios realizados en este sistema, Reséndez-Medina y Kobelkowsky (1991) registraron 44 especies, mientras que Juárez, Rojas-Galaviz, Mora Pérez y Zárate-Lomelí (2006) reportaron 42 especies y Díaz-Ruiz et al. (2008) 38 especies, por lo tanto, el número total registrado más las identificadas en el presente estudio ascendió a 77 especies para este sistema. De acuerdo con los resultados, se presentaron especies exclusivas durante cada año, destacando $C$. edentulus (2005), A. lamprotaenia (2006), M. atlanticus (2007) y Lutjanus cyanopterus (2008). Al respecto, se considera que un esfuerzo adicional de pesca en esta laguna resultaría en la captura de otras especies en función de otros ambientes como las zonas cercanas a la desembocadura de los ríos y la boca de conexión con el mar (Díaz-Ruiz et al., 2008). Las especies que habitan en el sistema La Mancha están adaptadas a la variación de los factores ambientales a través del tiempo, principalmente al gradiente de salinidad, el cual controla los movimientos de migración de las especies dentro y fuera del sistema en las diferentes épocas climáticas del año. Esto se reflejó en el mayor porcentaje de especies $(92 \%)$ del componente eurihalino (e.g. A. mitchilli, B. ronchus, D. rhombeus) y estenohalino (e.g. Gobionellus oceanicus, H. amblyrhynchus, Sphyraena barracuda, S. vomer). Estos resultados sugieren que los peces han desarrollado estrategias y rutas de migración para el uso de los hábitats lagunares-estuarinos como áreas de crianza, alimentación, desove y protección en alguna etapa de sus ciclos de vida con relación a las condiciones ambientales en espacio y tiempo (Díaz-Ruiz, Cano-Quiroga, Aguirre-León, \& Ortega-Bernal, 2004; Able, 2005; Cowan, Yáñez-Arancibia, Sánchez-Gil, \& Deegan, 2013).

En lagunas costeras tropicales, se conoce la utilización de variados hábitats por las especies de peces, determinada por respuestas complejas a los factores ambientales y biológi$\cos$, lo cual se refleja en las variaciones estacionales de la estructura comunitaria (Able, 2005; Aguirre-León et al., 2014). En este estudio se observaron cambios en los valores de diversidad y riqueza de especies relacionados con las condiciones hidrológicas y los diferentes 
ambientes del sistema, donde estos parámetros fueron más altos en la zona norte de la laguna donde predomina un ambiente polihalino. Este comportamiento fue similar a lo reportado por Castillo-Rivera et al. (2002) en la Laguna de Pueblo Viejo (Veracruz), donde la mayor diversidad y riqueza se presentó en la boca de comunicación con el Golfo, y donde las variaciones de salinidad jugaron un papel preponderante en la composición de peces; adicionalmente, otros estudios han indicado que el oxígeno disuelto, profundidad y turbidez también influyen en la diversidad de peces (Deegan et al. 1986; Barletta et al., 2005; Greenwood, Matheson, McMichael, \& MacDonald, 2007). Para el periodo 2005-2008 en La Mancha, los valores de diversidad y abundancia se relacionaron principalmente con las variaciones de salinidad, temperatura y profundidad durante cada época climática de la región. Autores como Thiel, Sepulveda, Kafemann y Nellen (1995), se refieren a estas variables como "controles" de los patrones temporales de la diversidad, distribución y composición de las especies en un sistema. En este sentido, se observó que la diversidad y riqueza fueron más altas al final de la temporada de lluvias e inicios de nortes (septiembre y noviembre), debido a la mayor abundancia de especies visitantes eurihalinas y estenohalinas del componente marino, que optimizan el uso del sistema, ligado a las variaciones estacionales de salinidad y al intercambio de especies con el mar durante las épocas climáticas de la región.

Las variaciones en los patrones de abundancia de las poblaciones también están influenciadas por los cambios en las condiciones ambientales del sistema y por los patrones de migración estacional de las especies durante sus ciclos de vida (Paperno, \& Brodie, 2004; Miller, \& Skilleter, 2006; Cowan, Grimes, \& Shaw, 2008). Así, la mayor densidad y biomasa de las especies, se registró principalmente en la zona norte de la laguna durante los meses de abril y junio (secas), cuando la salinidad y la temperatura fueron elevadas, lo cual sugiere que especies estenohalinas y eurihalinas se desplazan del mar hacia la laguna como juveniles y adultos, para crecer y alimentarse durante esta época como A. mitchilli, C. edentulus, D. rhombeus, E. melanopterus, $H$. amblyrhynchus y $S$. notata. Mientras que en el mes de julio (lluvias) los valores promedio más altos de estos parámetros se presentaron en la zona sur del sistema, donde individuos preadultos y adultos del componente estuarino, eurihalino y dulceacuícola, utilizan el sistema para alimentarse, madurar y/o protegerse como Sciades felis, C. aguadulce, C. parallelus, D. auratus, $M$. curema y $O$. aenigmaticum. Variaciones similares en la abundancia de estas especies de peces, también han sido observadas en otras lagunas costeras del Golfo de México por DíazRuiz, Pérez-Hernández y Aguirre-León (2003), Sosa-López, et al. (2012), Cowan et al. (2013), Aguirre-León et al. (2014).

En el sistema lagunar La Mancha existe una relación estrecha entre la abundancia de peces con los gradientes de salinidad, temperatura y profundidad. Esto se refleja en el evidente éxito ecológico de las especies dominantes, que, por su alta abundancia, frecuencia y distribución, han podido adaptar sus estrategias alimenticias, reproductivas, de crianza o protección, lo que ha favorecido su permanencia en el ecosistema (Lara-Domínguez et al., 2011; Aguirre-León et al., 2014). Tal es el caso de C. aguadulce, A. mitchilli, D. rhombeus y D. auratus que se consideraron como dominantes en el sistema lagunar en todos los años de muestreo. La especie C. aguadulce se distribuye como adulto preferentemente en la zona sur de la laguna, donde la salinidad y transparencia son menores por la descarga de los ríos Caño Grande y Sábalo, así, este bagre utiliza el sistema con fines tróficos y reproductivos durante lluvias. Cabe mencionar que la especie $C$. aguadulce ha sido citada anteriormente como C. melanopus (Marceniuk, \& Betancur, 2008). De esta manera, los resultados son similares con las observaciones realizadas en $C$. melanopus (sensu lato $C$. aguadulce) como especie dominante en otros sistemas lagunares del Golfo de México, donde ha sido la especie más abundante y con amplia distribución en áreas de mayor turbidez, menor salinidad e influencia fluvial, para fines 
reproductivos en los meses de lluvias y nortes (Yáñez-Arancibia, \& Lara-Domínguez, 1988; Ayala-Pérez, Ramos-Miranda, Flores-Hernández, Vega-Rodríguez, \& Moreno-Medina, 2008; Aguirre-León et al., 2010). La especie pelágica $A$. mitchilli, por su comportamiento eurihalino, estuvo relacionada con una salinidad variable, y presentó amplia distribución en áreas cercanas a la boca de conexión. Esta anchoa se capturó con mayor frecuencia durante los meses de estiaje, y realiza migraciones cortas entre áreas someras y más profundas del sistema para crianza y alimentación. Cowan et al. (2013) mencionaron que esta especie es más abundante durante las temporadas de secas en Laguna de Términos. Sin embargo, Castillo-Rivera, Zárate-Hernández, Ortiz-Burgos y Zavala-Hurtado (2010) señalaron que la mayor abundancia de esta especie se registró en época de lluvias para el sistema lagunar Pueblo Viejo al norte de Veracruz. Las mojarras D. rhombeus y D. auratus son especies con dependencia estuarina y gran capacidad eurihalina; en el presente estudio, se distribuyeron principalmente en áreas someras de manglar y de mayor salinidad, cercanas a la boca de conexión de La Mancha; además, realiza migraciones entre los ambientes del sistema, para alimentación y protección. D. rhombeus fue más abundante durante los meses de la temporada seca, mientras que $D$. auratus en los meses de lluvias, lo que mostró un comportamiento similar al de otras lagunas costeras estudiadas en el Golfo de México por Aguirre-León y Yáñez-Arancibia (1986); Aguirre-León y Díaz-Ruiz (2006); Aguirre-León et al. (2010).

El análisis de las especies dominantes reflejó sus adaptaciones en el uso del sistema durante sus ciclos de vida, se observó una alternancia estacional de especies relacionadas a los cambios ambientales que ocurren en el sistema a través del tiempo. Estos cambios se deben tanto al régimen de lluvias y al aporte fluvial, como a la influencia marina de una época climática a otra, lo que modifica las variables físicoquímicas de la laguna. Al respecto, otros estudios realizados en sistemas costeros tropicales también han mencionado un comportamiento similar sobre la influencia de los factores ambientales, como la salinidad, temperatura y profundidad, entre otros, en la estructura de la comunidad de peces (Bouchereau, Chaves, \& Monti, 2008; LaraDomínguez et al., 2011; Díaz-Ruiz et al., 2012; Cowan et al., 2013).

La mayoría de los sitios de muestreo se ubicaron en áreas con vegetación sumergida de $R$. maritima y circundante de $R$. mangle. Estas áreas actúan como áreas de protección y alimentación de alevines y juveniles de especies marinas y estuarinas de importancia ecológica y económica (Yáñez-Arancibia, Lara-Domínguez, \& Day, 1993; Bouchereau et al., 2008). Las zonas con vegetación tienen gran disponibilidad de alimento y mantienen poblaciones bénticas (Kanouse, La Peyre, \& Nyman, 2006; Vaslet, Bouchon-Navaro, Charrier, Louis, \& Bouchon, 2010), que a su vez son alimento para los peces y protegen a los juveniles de los depredadores. La mayoría de las especies de peces en estos sistemas tienen ciclos de vida típicamente estuarinos, con migraciones estacionales, que coinciden con los periodos de alta productividad y disponibilidad de alimento (Aguirre-León et al., 2014). Esto se reflejó en la composición trófica de los peces en La Mancha, ya que durante el periodo de estudio 2005-2008, predominaron especies consumidoras de primer y tercer orden en el sistema. Este comportamiento se relacionaría con la disponibilidad del alimento en el tiempo, las preferencias alimenticias de los peces o con la etapa del ciclo de vida de cada especie para utilizar el sistema (Rivera-Arriaga, Lara-Domínguez, Villalobos, \& Yáñez-Arancibia, 2003; Pineda-Peralta, Ramos-Miranda, Castillo-Rivera, \& Arreguín-Sánchez, 2016). Estudios realizados en otras lagunas del Golfo de México, reportan mayor abundancia de peces consumidores de segundo orden (YáñezArancibia, Lara-Domínguez, Aguirre-León, \& Díaz-Ruiz, 1986; Christensen \& Pauly, 1993). Este comportamiento difiere de lo encontrado para la laguna La Mancha, sin embargo, es necesario realizar mayor investigación sobre 
los hábitos alimenticios y relaciones tróficas de las especies de este sistema, ya que actualmente es muy escasa.

En la relación peces-hábitat se mostró que la salinidad, temperatura, oxígeno disuelto y profundidad, fueron las variables más importantes en la variación de la composición y distribución espacio temporal de las especies de peces en La Mancha. Dicho análisis permitió identificar la presencia de conjuntos de peces relacionados con los cambios físicoquímicos, donde la abundancia de las especies estuvo condicionada por las migraciones estacionales y por especies residentes del sistema. De esta manera, las especies asociadas con mayor influencia marina cercana a la boca de conexión fueron componentes estenohalinos y eurihalinos estacionales tales como $A$. surinamensis, $S$. notata, H. meeki, T. falcatus, $H$. brasiliensis, $E$. argenteus, $H$. amblyrhynchus, $C$. edentulus y $M$. curema, distribuyéndose en áreas someras con vegetación sumergida. El conjunto de especies asociadas a menores salinidades estuvo caracterizado por aquellas que toleran amplias variaciones de este parámetro, como C. aguadulce, A. mitchilli, D. auratus, D. rhombeus, E. plumieri y $B$. ronchus, que utilizan el sistema principalmente para alimentación, maduración y protección. Este comportamiento de grupos de peces también ha sido analizado por DíazRuiz et al. (2004), Greenwood et al. (2007), Bouchereau et al. (2008), Arceo-Carranza et al. (2010), Vaslet, Bouchon-Navaro, Charrier, Louis y Bouchon (2010).

El análisis de la información presentada permite establecer un evidente gradiente ambiental determinado por los procesos hidrológicos que ocurren en el sistema, y que están relacionados con los cambios estacionales de la región. De la misma manera, los valores de diversidad, abundancia y frecuencia de la ictiofauna responden a ese gradiente en espacio y tiempo. Esta información, también nos permite entender los procesos ecológicos que ocurren en ecosistemas similares e identificar hábitats prioritarios de conservación y hacer uso adecuado de los recursos naturales. Este estudio constituye un punto de partida para evaluaciones posteriores, y una herramienta de monitoreo del estado de salud ambiental de esta laguna, a fin de contribuir a la conservación de su biodiversidad y protección como área importante para la crianza, alimentación, protección y/o reproducción de recursos pesqueros en este sitio con categoría Ramsar.

\section{AGRADECIMIENTOS}

Al Departamento de Hidrobiología de la Universidad Autónoma Metropolitana Iztapalapa (Proyecto CBS-147.05.007) y al CONACyT (FOMIX) C01-37014 el apoyo institucional y económico para realizar este estudio. Los autores agradecen a Celerino Barradas su apoyo en los muestreos de campo y a todos los estudiantes su colaboración en campo y laboratorio. Se agradece a los árbitros sus comentarios y sugerencias realizadas.

\section{RESUMEN}

La laguna La Mancha en el Golfo de México, es un sistema costero con categoría Ramsar por su alta biodiversidad, numerosas especies de peces dependen de el para realizar sus ciclos de vida. Fue analizada la variación espacial y temporal de la diversidad, abundancia y asociaciones de peces y su relación con los factores fisicoquímicos. Entre abril de 2005 y enero de 2008, se realizaron 95 muestreos diurnos en siete sitios donde se registraron las características del agua y la composición de la comunidad de peces. El sistema presentó variaciones espacio temporales de los parámetros físicoquímicos, definiendo ambientes contrastantes de acuerdo con los gradientes locales. Se recolectaron 5974 individuos, pertenecientes a 50 especies, 39 géneros y 25 familias, cuatro especies son registros nuevos para el sistema. La diversidad y riqueza de especies fueron altas en el ambiente salobre y bajos en zonas dulceacuícolas. En septiembre (lluvias) y noviembre (nortes) la diversidad $\left(H^{\prime}=1.52\right)$ y riqueza $(D=1.99)$ fue más alta. La abundancia de los peces fue mayor hacia la boca de conexión con el mar. La densidad y biomasa (1.17 indiv. $/ \mathrm{m}^{2}, 4.33 \mathrm{~g} / \mathrm{m}^{2}$ ) fue mayor en abril (secas) y el peso promedio (30.82 $\mathrm{g}$ /ind) en julio (lluvias). El Índice de Valor de Importancia (IVI) definió cuatro especies dominantes que representan el $75 \%$ de la abundancia numérica y el $46 \%$ de la captura total en peso. El Análisis de Correspondencia Canónica (ACC) mostró que la relación peces-hábitat fue explicada en un $57 \%$ de la varianza total para los primeros dos ejes, donde las variaciones de temperatura, salinidad, oxígeno disuelto y profundidad fueron 
los factores ambientales que determinaron la composición, distribución, componentes comunitarios y categorías tróficas de los peces en la comunidad. Este estudio aporta información sobre la relación del ambiente con la estructura de la comunidad íctica de La Mancha, y representa una base para realizar monitoreo ecológico que permita incrementar el conocimiento necesario sobre el ecosistema y sus poblaciones de peces, con la finalidad de proponer estrategias de manejo para la conservación de los recursos pesqueros de Veracruz.

Palabras clave: factores fisicoquímicos, estructura comunitaria, dominancia, estructura trófica, relaciones peceshábitat, La Mancha, México.

\section{REFERENCIAS}

Able, K. W. (2005). A re-examination of fish estuarine dependence: Evidence for connectivity between estuarine and ocean habitats. Estuarine Coastal and Shelf Science, 6, 5-17.

Aguirre-León, A., \& Yáñez-Arancibia, A. (1986). Las mojarras de la Laguna de Términos, Campeche: Taxonomía, biología, ecología y dinámica trófica (Pisces: Gerreidae). Anales del Instituto de Ciencias del Mar y Limnología, Universidad Nacional Autónoma de México, 13, 369-444.

Aguirre-León, A., \& Díaz-Ruiz, S. (2006). Estructura de tallas, madurez gonádica y alimentación del pez Diapterus rhombeus (Gerreidae) en el sistema fluviodeltaico Pom-Atasta, Campeche, México. Revista de Biología Tropical, 54, 599-611.

Aguirre-León, A., Díaz-Ruiz, S., \& Ramírez-Huerta, A. (2010). Ecología de peces dominantes costeros tropicales. Estudio para el sistema fluvio-deltaico PomAtasta, Campeche. Serie Académicos 96. México: CBS, UAM-Xochimilco.

Aguirre-León, A., Pérez-Ponce, H. E., \& Díaz-Ruiz, S. (2014). Heterogeneidad ambiental y su relación con la diversidad y abundancia de la comunidad de peces en un sistema costero del Golfo de México. Revista de Biología Tropical, 62, 145-163.

Arceo-Carranza, D., Vega-Cendejas, M. A., MonteroMuñoz, J. L., \& Hernández de Santillana, M. J. (2010). Influencia del hábitat en las asociaciones nictimerales de peces en una laguna costera tropical. Revista Mexicana de Biodiversidad, 81, 823-837.

Ayala-Pérez, L. A., Ramos-Miranda, J., Flores-Hernández, D., Vega-Rodríguez, B., \& Moreno-Medina, U. (2008). Biological and ecological characterization of the catfish Cathorops melanopus (Gunther 1864) off the coast of Campeche, Mexico. Ciencias del Mar, 34, 453-465.
Barletta, M., Barletta-Bergan, A., Saint-Paul, U., \& Hubold, G. (2005). The role of salinity in structuring the fish assemblages in a tropical estuary. Journal of Fish Biology, 66, 45-72.

Bouchereau, J. L., Chaves, P., \& Monti, D. (2008). Factors structuring the ichthyofauna assemblage in a mangrove lagoon (Guadeloupe, French West Indies). Journal of Coastal Research, 24, 969-982.

Castillo-Rivera, M., Zavala-Hurtado, A., \& Zárate- Hernández, R. (2002). Exploration of spatial and temporal patterns of fish diversity and composition in a tropical estuarine system of Mexico. Reviews in Fish Biology and Fisheries, 12, 167-177.

Castillo-Rivera, M., Zárate-Hernández, R., Ortiz-Burgos, S., \& Zavala-Hurtado, A. (2010). Diel and seasonal variability in the fish community structure of a mud bottom estuarine habitat in the Gulf of Mexico. Marine Ecology, 31, 633-642.

Castro Aguirre, J. L., Espinoza-Pérez, H., \& SchmitterSoto, J. J. (1999). Ictiofauna estuarino-lagunar y vicaria de México. México: Limusa.

Christensen, V., \& Pauly, D. (1993). Trophic models of aquatic ecosystem. ICES Theme Session, DANIDA, ICLARM Conference Proceeding. Manila Philippines: International Center for Living Aquatic Resources Management.

Contreras-Espinosa, F., Castañeda-López, O., \& RiveraGuzmán, N. (2006). La laguna. En P. Moreno-Casasola (Ed.), Entornos veracruzanos: La costa de La Mancha (pp.151-167). México: INECOL.

Cowan, J. H., Grimes, C. B., \& Shaw, R. F. (2008). Life history, history, hysteresis, and habitat change in Louisiana's coastal ecosystem. Bulletin of Marine Science, 83, 197-215.

Cowan, J. H., Yáñez-Arancibia, A., Sánchez-Gil, P., \& Deegan L. (2013). Estuarine nekton. En J. W. Day, B. C. Crump, W. M. Kemp, \& A. Yáñez-Arancibia (Eds.), Estuarine Ecology (pp. 327-355). Nueva Jersey: Willey \& Sons.

Deegan, L., Day, J. W., Gosselink, J. G., Yáñez-Arancibia, A., Chávez, G., \& Sánchez-Gil, P. (1986). Relationship among physical characteristics, vegetation distribution and fisheries yield in Gulf of Mexico estuaries. En D.A. Wolfe (Ed.), Estuarine variability (pp. 83-100). Nueva York: Academic Press.

De la Cruz-Agüero, G. (1994). ANACOM Sistema para el análisis de comunidades. Versión 3.0. México: CICIMAR-IPN

De la Lanza Espino, G., Rodríguez, I. P., Baus, S. C., \& Pulido, S. H. (2011). Química de las lagunas costeras. En G. de la Lanza Espino, \& S. H. Pulido (Eds.), Ambiente, biología, sociedad, manejo y legislación 
de sistemas costeros mexicanos (pp. 141-177). México: UMSNH, WWF, Plaza y Valdés.

Díaz-Ruiz, S., Pérez-Hernández, M., \& Aguirre-León, A. (2003). Characterization of fish assemblages in a tropical coastal lagoon in the northwest Gulf of Mexico. Ciencias Marinas, 29, 631-644.

Díaz-Ruiz, S., Cano-Quiroga, E., Aguirre-León, A., \& Ortega-Bernal, R. (2004). Diversidad, abundancia y conjuntos ictiofaunísticos del sistema lagunar-estuarino Chantuto-Panzacola, Chiapas, México. Revista de Biología Tropical, 52, 187-199.

Díaz-Ruiz, S., Aguirre-León, A., Calva-Benítez, L. G., \& Barba-Macías, E. (2012). Comportamiento ambiental y estructura comunitaria de peces en el sistema Carretas-Pereyra, Reserva de la Biosfera La Encrucijada, Chiapas. En A. J. Sánchez, X. Chiappa-Carrara, \& R. Brito-Pérez (Eds.), Recursos acuáticos costeros del sureste (pp. 415-434). México: CONCIYTEY-UNAM.

Díaz-Ruiz, S., Aguirre-León, A., Juárez, M., Matsumoto, J., \& Hernández, J. A. (2008). Evaluación ecológica de las comunidades de peces: Laguna La Mancha y Laguna el Ostión, Veracruz Golfo de México. (Informe Final-CONACYT-FOMIX-37014). Veracruz: Instituto de Ecología y Universidad Autónoma Metropolitana.

Ficha Informativa de los Humedales de Ramsar (FIR), (2003). La Mancha-El Llano. Xalapa, Veracruz, México.

Fischer, W. (1995). Species identification sheets for fishery purpose. Western Central Atlantic (Fishing area 31). Vol. 1-5. Rome: FAO.

Froese, R., \& Pauly, D. (2017). FishBase. Versión 02/2017. Recuperado de http://www.fishbase.org

García, E. (2004). Modificaciones al sistema de clasificación climática de Köppen. México: Instituto de Geografía, UNAM.

Greenwood, M. F. D., Matheson, Jr. R. E., McMichael, Jr. R. H., \& MacDonald, T. C. (2007). Community structure of shoreline nekton in the estuarine portion of the Alafia River, Florida: Differences along a salinity gradient and inflow-related changes. Estuarine, Coastal and Shelf Science, 74, 223-238.

Hair, J. F., Anderson, R. E., Tatham, R. L., \& Black, W.C. (2001). Multivariate data analysis. Madrid, España: Prentice Hall.

Juárez, E. A., Rojas Galaviz, J. L., Mora Pérez, C., \& Zárate Lomelí, D. (2006). Los peces. In P. Moreno-Casasola (Ed.), Entornos veracruzanos: La costa de La Mancha (pp.327-340). Veracruz, México: INECOL.

Kanouse, S., La Peyre, M. K., \& Nyman J. A. (2006). Nekton use of Ruppia maritima and non-vegetated bottom habitat types within brackish marsh ponds. Marine Ecology Progress Series, 327, 61-69.

Lara-Domínguez, A. L., Day, J. W., Yáñez-Arancibia, A., \& Sainz-Hernández, E. (2006). A dynamic characterization of water flux through a tropical ephemeral inlet, La Mancha Lagoon, Gulf of Mexico. En V.P. Singh, \& Y. Ju-Xu (Eds.), Coastal hydrology and processes (pp. 413-422). Colorado: Water Resources Publication.

Lara-Domínguez, A. L., Franco-López, J., Bedia-Sánchez, C., Abarca-Arenas, L. G., Díaz-Ruiz, S., AguirreLeón, A., González-Gándara, C., \& Castillo-Rivera, M. (2011). Diversidad de peces en los ambientes costeros y plataforma continental. En A. Cruz-Angón (Ed.), La biodiversidad en Veracruz: estudio de estado (pp. 505-516). México: CONABIO, UV, INECOL, Gobierno del Estado de Veracruz.

Levene, H. (1960). Robust test for equality on variance. En I. Olkin, S. G. Ghurye, W. Hoeffding, W. G. Madow, \& H. B. Mann (Eds.), Contributions of probability and statistics (pp. 278-292). California: Stanford University.

López-Ortega, M., Pulido, G., Serrano, A., Gaytán, J. C., Monks, W. S., \& López, M. (2010). Evaluación estacional de las variables físicoquímicas del agua de la Laguna de Tampamachoco, Veracruz, México. Revista Científica UDO Agrícola, 12, 713-719.

Marceniuk, A. P., \& Betancur-R, R. (2008). Revision of the species of the genus Cathorops (Siluriformes: Ariidae) from Mesoamerica and the Central American Caribbean, with description of three new species. Neotropical Ichthyology, 6, 25-44.

Margalef, R. (1969). Perspectives in ecological theory. Chicago: University of Chicago.

McGarigal, K., Cushman, S., \& Stafford, S. (2000). Multivariate statistics for wildlife and ecology research. Nueva York: Springer-Verlag.

Miller, S. J., \& Skilleter, G. A. (2006). Temporal variation in habitat use by nekton in a subtropical estuarine system. Journal of Experimental Marine Biology and Ecology, 337, 82-95.

Nelson, J. S. (2006). Fishes of the world. Nueva Jersey: Willey \& Sons.

Paperno, R., \& Brodie, R. B. (2004). Effects of environmental variables upon the spatial and temporal structure of a fish community in a small, freshwater tributary of the Indian River Lagoon, Florida. Estuarine, Coastal and Shelf Science, 61, 229-241.

Pielou, E. C. (1966). The measurement of diversity in different types of biological collections. Journal of Theoretical Biology, 13, 131-144. 
Pineda-Peralta, A., Ramos-Miranda, J., Castillo-Rivera, M., \& Arreguín-Sánchez, F. (2016). Cambios en la dieta de dos especies de mojarras (Gerreidae) en la Laguna de Términos, Campeche, México. Revista de Biología Marina y Oceanografia, 51, 385-394.

Reséndez-Medina, A. \& Kobelkowsky-Díaz, A. (1991). Ictiofauna de los sistemas lagunares costeros del Golfo de México. Universidad y Ciencia, 8, 91-110.

Rivera-Arriaga, E., Lara-Domínguez, A. L., Villalobos, G., \& Yáñez-Arancibia, A. (2003). Trophodynamic ecology of two critical habitats (seagrass and mangrove) in Terminos lagoon, southern Gulf of Mexico. En F. Arreguín, D. Pauly, \& D. Zeeler (Eds.), ECOPHATHII Workshop Proceeding (pp. 245-254). Vancouver, Canada: University British Columbia.

Shannon, E. C., \& Wiener, W. (1963). The mathematical theory of communication. Illinois: University of Illinois.

Snedden, G. A., Cable, J. E., \& Kjerfve, B. (2013). Estuarine geomorphology and coastal hidrology. En J. W. Day, B. C. Crump, W. M. Kemp, \& A. Yáñez-Arancibia (Eds.), Estuarine ecology (pp. 19-38). Nueva Jersey: Willey \& Sons.

Sosa-Pérez, A., J. Ramos-Miranda, L. Ayala, D. FloresDomínguez, G. Villalobos \& F. Gómez. (2012). Cambios de largo plazo en la comunidad de peces y variables fisicoquímicas en la Laguna de Términos, sur del Golfo de México. En A. J. Sánchez, X. Chiappa-Carrara, \& R. Brito-Pérez (Eds.), Recursos acuáticos costeros del sureste (pp. 307-330). México: CONCIYTEY-UNAM.

Ter Braak, C. J. F., \& Verdonschot, P. F. M. (1995). Canonical correspondence analysis and related multivariate methods in aquatic ecology. Aquatic Science, 57, 255-289.

Thiel, R., Sepulveda, A., Kafemann, R., \& Nellen, W. (1995). Environmental factors as forces structuring the fish community of the Elbe estuary. Journal of Fish Biology, 46, 47-69.

Torres-Alvarado, M. R., Calva-Benítez, L. G., ÁlvarezHernández, S., \& Trejo-Aguilar, G. (2016). Anaerobic microbiota: spatial-temporal changes in the sediment of a tropical coastal lagoon with ephemeral inlet in the Gulf of Mexico. Revista de Biología Tropical, 64, 1759-1770.

Vaslet, A., Bouchon-Navaro, Y., Charrier, G., Louis, M., \& Bouchon, C. (2010). Spatial patterns of mangrove shoreline fish communities in relation with environmental variables in Caribbean lagoons. Estuaries and Coasts, 33, 195-210.

Yáñez-Arancibia, A. (1986). Ecología de la zona costera, análisis de siete tópicos. México: AGT Editor.

Yáñez-Arancibia, A., \& Lara-Domínguez, A. L. (1988). Ecology of three sea catfishes (Ariidae) in a tropical coastal ecosystem southern Gulf of Mexico. Marine Ecology Progress Series, 49, 215-230.

Yáñez-Arancibia, A., Lara-Domínguez, A. L., AguirreLeón, A., Díaz-Ruiz, S., Amezcua Linares, F., Flores, D., \& Chavance, P. (1985). Ecology of dominant fish population in tropical estuaries: Environmental factors regulating biological strategies and production. En A. Yáñez-Arancibia (Ed.), Fish community ecology in estuaries and coastal lagoons: towards an ecosystem integration (pp. 311-366). México: Editorial Universitaria-UNAM.

Yáñez-Arancibia, A., Lara-Domínguez, A. L., AguirreLeón, A., \& Díaz-Ruiz, S. (1986). Feeding ecology of tropical estuarine fishes in relation recruitment processes. En Workshop on Recruitment in Tropical Coastal Demersal Communities (pp. 73-94). IOC/ FAO Report No. 44-Supplement.

Yáñez-Arancibia, A., Lara-Domínguez, A. L., \& Day, J. W. (1993). Interactions between mangrove and seagrass habitats mediated by estuarine nekton assemblages: coupling of primary and secondary production. Hydrobiologia, 264, 1-12.

Zar, J. H. (1999). Biostatistical analysis. Nueva Jersey: Prentice-Hall.

Zárate-Hernández, R., Castillo-Rivera, M., SanvicenteAñorve, L., \& Ortiz-Burgos, S. (2012). Cambios espaciales, nictímeros y estacionales en la estructura de la comunidad de peces en un estuario tropical mexicano. Ciencias Marinas, 38, 665-676. 